\title{
Abstracts from the 33rd Annual Scientific Meeting of the Canadian Geriatrics Society Toronto, April 2013
}

DOI: http://dx.doi.org/10.5770/cgj.16.85

The opinions expressed in the abstracts are those of the authors and are not to be construed as the opinion of the publisher (Canadian Geriatrics Society) or the organizers of the 33rd Annual Scientific Meeting of the Canadian Geriatrics Society. Although the publisher (Canadian Geriatrics Society) has made every effort to accurately reproduce the abstracts, the Canadian Geriatrics Society and the 33rd Annual Scientific Meeting of the Canadian Geriatrics Society assumes no responsibility and/or liability for any errors and/or omissions in any abstract as published.

\section{ORAL PRESENTATIONS AT THE 33 RD ANNUAL SCIENTIFIC MEETING OF THE CANADIAN GERIATRICS SOCIETY}

\section{TRAINEE POSTER ABSTRACTS}

\begin{abstract}
Medical Characteristics of the "Oldest Old": a Descriptive Epidemiological Study of 85+-Year-Old Patients in an Academic Primary Care Centre in Toronto

C. Tsoi ${ }^{1}$, J. Nie ${ }^{2}$, S. Tracy ${ }^{2}$, L. Wang ${ }^{2}$, R. Upshur ${ }^{1}$, K. Choi ${ }^{1}$, H-W. $\mathrm{Li}^{1}$, J. Chow ${ }^{3}$

${ }^{1}$ Department of Family and Community Medicine, Sunnybrook Health Sciences Centre, Toronto, ON; ${ }^{2}$ Primary Care Research Unit, Sunnybrook Health Sciences Centre, Toronto, ON; ${ }^{3}$ Faculty of Medicine, University of Toronto, Toronto, ON, Canada
\end{abstract}

Background/Purpose: The 85+-year-old population - the "oldest old" - is now the fastest growing age segment in Canada. Although existing research demonstrates high health services utilization and prescribed medications in this population, little epidemiological evidence is available to guide care for this age group.

Objective: To describe the epidemiological characteristics of common health conditions and medication prescriptions in the "oldest old".

Methods: We conducted a retrospective chart review of all family practice patients aged $\geq 85(\mathrm{~N}=564$; 209M:355F) at Sunnybrook Health Sciences Centre, Toronto. Electronic medical records were reviewed for all current chronic conditions and medication prescriptions, and then stratified by sex and age subgroup (85--89, 90-94, $95+$ ) for descriptive analysis.
Results: On average, patients experienced 6.4 concurrent chronic conditions and took 6.8 medications. Most conditions were related to cardiovascular (79\%) and bone health (65\%). Hypertension (65\%) was the most common condition. Bonerelated conditions (e.g., osteoarthritis, osteoporosis) and hypothyroidism predominantly affected women $(p<.001)$, while coronary artery disease and T2DM were more prevalent in men $(p<.05)$. The top two prescribed medications were Atorvastatin (33\%) and ASA $81 \mathrm{mg}(33 \%)$. Males were more likely to be prescribed lipid-lowering medications, while females were more likely to receive osteoporosis therapy $(p<.001)$. Patients received less lipid-lowering therapy with increasing age $(p<.001)$.

Discussion \& Conclusion: Multimorbidity and polypharmacy are highly prevalent in patients over 85 . Most clinical concerns in this population relate to cardiovascular and bone health; medications predominantly treat risk factors. In the absence of epidemiological data to guide clinical decisionmaking, this study provides a first look at the common health concerns and medication profiles in this population.

\section{Cognitive Inhibition and Decision-Making in Elderly} Suicidal Behaviour

S. Richard-Devantoy ${ }^{1,2}$, F. Jollant ${ }^{1}$, G. Turecki ${ }^{1}$

${ }^{1}$ Department of Psychiatry \& Douglas Mental Health University Institute, McGill Group for Suicide Studies, McGill 
University, Montréal, QC, Canada; ${ }^{2}$ Laboratoire de Psychologie des Pays de la Loire EA 4638, Université de Nantes et Angers, France

Background/Purpose: Although a serious public health concern, very little is known about the neurocognitive basis of suicidal behaviour in the elderly. Here we aimed at: 1) assessing alterations in cognitive inhibition in suicidal depressed elderly people, and 2) reviewing the literature on cognitive inhibition and decision-making in elderly suicidal behaviour.

Methods: First, we compared 20 currently depressed patients, aged 65 and older, who had recently attempted suicide to 20 elderly subjects with a current depression but no personal history of suicide attempts and 20 elderly controls. Different aspects of cognitive inhibition were examined: access to relevant information (reading with distraction task), suppression of no longer relevant information (Trail Making Test, Rule Shift Cards), and restraint of cognitive resources to relevant information (Stroop Test, Hayling Sentence Completion Test, Go/No-Go task). Second, systematic MEDLINE literature search was performed on neurocognitive deficits in suicidal behaviour. References from our research group's online database were also selected (http://www.bdsuicide. disten.com).

Results: After adjustment for age, depression intensity, Mini-Mental Status Examination score, and speed of information processing, suicidal depressed elderly people showed significant impairments in all three domains of cognitive inhibition in comparison to the affective and healthy control groups. Moreover, the results of a meta-analysis study will also be presented.

Discussion \& Conclusion: Cognitive inhibition deficits and impaired decision-making appear to be part of a series of cognitive deficits and may impair the patient's capacity to respond adequately to stressful situations, which could subsequently lead to an increased risk of suicidal behaviour during late-life depression. Suicide prevention interventions may be developed to specifically target cognitive impairment in depressed elderly people.

Methodological Challenges in Determining the Causal Association Between Anticholinergic Drug Use and Memory Impairment in Older Adults

M. Kashyap ${ }^{1}$, S. Belleville ${ }^{1}$, B. Mulsant ${ }^{2}$, S. Hilmer ${ }^{3}$, C. Tannenbaum ${ }^{1}$

${ }^{1}$ Centre de Recherche, Institut Universitaire de Gériatrie de Montréal, Université de Montréal, Montreal, QC, Canada; ${ }^{2}$ Centre for Addiction and Mental Health, University of
Toronto, Toronto, ON, Canada; ${ }^{3}$ University of Sydney, Sydney, NSW, Australia

Background/Purpose: Anticholinergic drugs may induce cognitive decline in older adults, but data are conflicting. One research challenge is ascertaining the effect of different exposure \& outcome definitions on measures of association.

Methods: Using baseline and 1-year follow-up data from 131 community patients aged $60+$, we applied 4 measures of anticholinergic drug exposure (the Anticholinergic Drug Burden Index (DBI), ACB, ADS \& ARS, and 4 definitions of cognitive decline (neuropsychological test raw change scores, the RCI, the standardized regression based change score (SRB), and the clinical diagnosis of a new mild neurocognitive disorder according to DSM-5 criteria). The frequency of classification for each patient and the number needed to harm $(\mathrm{NNH})$ was calculated according to each exposure \& outcome definition. The consistency of associations between drug exposure \& cognitive decline was examined using logistic regression models for each definition.

Results: The Anticholinergic DBI identified the smallest number of patients with an increase in anticholinergic exposure $(n=18)$ and the ACB identified the largest number $(n=$ 23). The RCI identified cognitive decline in only 6 patients; 12 patients were diagnosed with a new mild neurocognitive disorder, 44 had changes in raw neuropsychological test scores, and 99 had changes on the SRB measure. The NNH ranged from 0-100. A significant association between increased anticholinergic drug exposure $\&$ cognitive decline was found in only one model that used the Anticholinergic DBI and the SRB measure of cognitive decline on the Trail B test (OR $2.2 ; 95 \%$ CI $-1.1-8.06)$.

Discussion \& Conclusion: The choice of definition by which to classify drug exposure and cognitive decline has a significant effect on the results of causal association studies.

Is a Multifaceted Knowledge Translation Intervention Feasible and Acceptable in Long-Term Care? A Qualitative Study with ViDOS Participants

C. Kennedy ${ }^{1}$, L. Lohfeld ${ }^{2}$, J.D. Adachi ${ }^{1}$, S. Morin ${ }^{3}$, S. Marr ${ }^{1}$, R.G. Crilly ${ }^{4}$, R.G. Josse ${ }^{5}$, G.Ioannidis ${ }^{1}$, L.M. Giangregorio ${ }^{6}$, L. Thabane ${ }^{2}$, A. Papaioannou ${ }^{1}$

${ }^{1}$ Department of Medicine, McMaster University, Hamilton, ON; ${ }^{2}$ Department of Clinical Epidemiology \& Biostatistics, McMaster University, Hamilton, ON; ${ }^{3}$ Department of Medicine, Division of General Internal Medicine, McGill University, Montreal, QC; ${ }^{4}$ Schulich School of Medicine \& Dentistry, The University of Western ON, London, ON; ${ }^{5}$ Department of Medicine, Division of Endocrinology and 
Metabolism, University of Toronto, Toronto, ON; ${ }^{6}$ Department of Kinesiology, Faculty of Applied Health Sciences, University of Waterloo, Waterloo, ON, Canada

Background/Purpose: Few studies in long-term care (LTC) have examined the feasibility and acceptability of knowledge translation (KT) programs. We conducted a qualitative evaluation of LTC professionals' experience with a multifaceted, interdisciplinary KT intervention.

Methods: We invited Medical Directors, Directors of Care (Nursing), and Consultant Pharmacists who participated in the Vitamin D and Osteoporosis Study (ViDOS), a randomized controlled trial conducted in 40 Ontario LTC homes (19 intervention, 21 control). ViDOS objectives were to evaluate the feasibility and effectiveness of a KT model to increase the use of osteoporosis/fracture prevention strategies. Multifaceted components included: 3 webinar presentations by expert opinion leaders, audit \& feedback, point-of-care tools, internal champions, and action planning for quality assurance. In this qualitative evaluation study, we conducted individual, semi-structured telephone interviews and analyzed transcripts using thematic framework analysis.

Results: Overall, 4 Directors of Care, 7 Consultant Pharmacists, and 2 Medical Directors participated. Medical Directors were not included in group comparisons due to the limited sample size. Most respondents (10/13) attended all sessions and thought it was a valuable experience. The on-site involvement of an expert opinion leader was seen as most useful by all participant groups. Perceived utility of the other KT components varied by group: Directors of Care highly valued audit \& feedback, whereas Consultant Pharmacists highly valued small-group learning and internally nominated champions. Common themes for improvement were ready-touse educational fact sheets and having expert opinion leaders attend in person or via video conference.

Discussion \& Conclusion: The ViDOS intervention was well-received by study participants we interviewed. Lessons learned in this study can inform future KT initiatives in LTC.

Bone Health in Prostate Cancer Survivors Receiving Androgen Deprivation Therapy (ADT): Examining Enablers and Barriers to Care

K. Bies ${ }^{1}$, J.M. Jones ${ }^{2}$, P. Catton ${ }^{2}$, P. Warde 2 , N. Fleshner ${ }^{2}$, A. Matthew $^{2}$, S.M.H. Alibhai ${ }^{3}$

${ }^{1}$ University of Toronto, Toronto, ON; ${ }^{2}$ University Health Network, Princess Margaret Cancer Centre, Toronto, ON, ${ }^{3}$ University Health Network, Toronto General Hospital, Toronto, ON, Canada
Background/Purpose: Older men receiving ADT for prostate cancer have a 5-10 fold increased rate of bone loss and up to $20 \%$ fracture risk by 5 years of treatment. Guidelines exist for bone-loss management in this population, but adherence is poor. We assessed the knowledge and current practices regarding bone-loss management in a sample of Canadian prostate cancer $(\mathrm{PC})$ specialists.

Methods: Using Dillman's tailored design method, a questionnaire was distributed to Canadian PC specialists through three major specialty organizations.

Results: 156 PC specialists completed the questionnaire. Awareness of recommendations for frequency of repeat bone mineral density (BMD) scans $(76.3 \%)$ and vitamin D use $(70.3 \%)$ was relatively high, but lower for calcium intake $(53.2 \%)$ and amount of weekly exercise (20.7\%). A minority were aware of the true prevalence of osteoporosis in otherwise healthy 60 -year-old males $(27.3 \%)$, the risk of developing osteoporosis after 1 year of continuous ADT (37.8\%), and the excess fracture risk after 5 years on ADT (14.7\%). 34.4\% of respondents reported routinely ordering BMD tests pre-ADT treatment and $36.6 \%$ ordered routine BMD tests after initiating ADT. Most reported routinely recommending exercise, calcium, and supplemental vitamin D. The most significant barriers to implementing the recommendations were lack of time to counsel patients and lack of supporting structures (e.g., patient education).

Discussion \& Conclusion: Participants were fairly knowledgeable regarding recommendations for managing bone loss in men on ADT. However, there were gaps in knowledge regarding risk of developing osteoporosis and in clinical surveillance and risk assessment. These findings suggest the need for knowledge translation strategies and tools to address this gap between evidence and clinical practice.

\section{An Audit on the Justification of Invasive Treatment in Elderly Patients}

S. Kanji

St George's University of London, London, UK

Background/Purpose: An audit was conducted on the recorded reason for invasive treatments in older patients. According to the British Geriatric Society and NICE guidelines catheterisation and regular sedation should be avoided in elderly patients especially those with delirium. Additionally, many studies have been conducted showing a link between sedation and delirium. The aim of the study was to discern whether invasive treatments such as the use of catheters, cannulas, intravenous antibiotics, and the provision of sedatives is justified, as these procedures have associated risks including delirium. 
Methods: Data were collected data from three Geriatric Medicine wards, looking at the first 48 hours of care. Data were assembled on patient demographics, patient's AMT score, invasive procedures conducted, and the reason for the procedure. The gold standard for this audit is that $100 \%$ of procedures are provided with a reason in the notes.

Results: $72 \%$ of patients were Caucasians, the mean age $84.6 \pm 8.0(\mathrm{SD})$, and $50 \%$ of patients in the audit were classed as delirious. The findings show that $98 \%$ of invasive procedures were not clearly justified in the notes, regardless of whether the patient was suffering from delirium. $97 \%$ of cannulas inserted were not justified in the notes and was the most common invasive procedure.

Discussion \& Conclusion: These results are in agreement with the hypothesis that the majority of procedures will not have a clear justification in the notes. A justification column could be added in order to make doctors think twice about their reasoning for providing these treatments and thus prompt doctors to provide a reason for these invasive procedures.

\section{Co-Morbidity in the Very Old Type 2 Diabetic Patient}

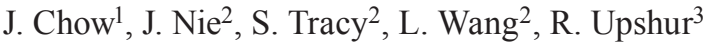 \\ ${ }^{1}$ Faculty of Medicine, University of Toronto, Toronto, ON; ${ }^{2}$ Primary Care Research Unit, Sunnybrook Health Sciences Centre, Toronto, ON; ${ }^{3}$ Department of Family and Community Medicine, Sunnybrook Health Sciences Centre, Toronto, ON, Canada}

Background/Purpose: The management of multimorbidity in the oldest old (aged $\geq 85$ ) is recognized as one of the most pressing challenges facing clinicians. Given the increasing prevalence of T2DM in this population, a more precise understanding of the epidemiology of co-existing chronic illnesses is necessary to guide therapy.

Objective: To characterize co-morbidity in T2DM patients aged $\geq 85$ in primary care.

Methods: We conducted a retrospective chart review of family practice patients aged $\geq 85$ at Sunnybrook Health Sciences Centre, Toronto. Electronic medical records were reviewed for all chronic conditions. For all T2DM patients, each condition was coded as "concordant/discordant" with diabetic care (whether it is related to its pathophysiologic risk profile or management complications), "symptomatic/ asymptomatic" (whether it causes symptoms noticeable to the patient), and "clinically dominant/not dominant" (complex or serious enough to eclipse the management of all other health conditions). We recorded the total number of co-morbid conditions (other than diabetes) in each category for each patient.
Results: $\quad$ T2DM patients comprised 16\% $(\mathrm{n}=91 ; 42 \mathrm{M}: 49 \mathrm{~F})$ of all patients aged $\geq 85$. On average, each patient experienced 6.8 co-morbid conditions other than diabetes (range: $2-16)$; patients generally had discordant and symptomatic co-morbidities $(p<.001) .47 \%(\mathrm{n}=43)$ of our sample had at least one clinically dominant condition.

Discussion \& Conclusion: Co-morbidity is highly prevalent in very old type 2 diabetic patients. Most co-morbid chronic conditions are symptomatic and discordant with diabetes care. A significant proportion of these patients also suffer from clinically dominant conditions. In the absence of evidence-based care guidelines for this age group, it may be beneficial to focus therapy on the management of symptoms and functional limitations rather than aggressively pursuing risk factor modification.

The Impact of Bone Mineral Density, Fracture Assessment, and Osteoporosis Education in Men Treated by Androgen Deprivation for Prostate Cancer

M. Nadler ${ }^{1}$, S. Alibhai ${ }^{2}$, P. Catton ${ }^{3}$, C. Catton ${ }^{1}$, J. Jones ${ }^{1}$

${ }^{I}$ Department of Family and Community Medicine, Princess Margaret Cancer Centre, Cancer Survivorship Program, Toronto, ON; ${ }^{2}$ Department of Medicine, University Health Network, Toronto, ON; ${ }^{3}$ Radiation Oncology, Princess Margaret Cancer Centre, Toronto, ON, Canada

Background/Purpose: Men receiving androgen deprivation therapy for prostate cancer have low knowledge of osteoporosis (OP) and are engaging in few healthy bone behaviours (HBBs). A multi-component intervention was piloted in this population, and changes in OP knowledge, self-efficacy, health beliefs, and engagement in HBBs were evaluated.

Methods: A pre-post pilot study was performed in a convenience sample of men recruited from the genitourinary clinics at Princess Margaret Hospital. Men were sent personalized letters explaining their dual X-ray absorptiometry (DXA) results and fracture risk assessment with an OP-related education booklet. Participants completed questionnaires assessing OP knowledge, self-efficacy, health beliefs, and current engagement in HBBs at baseline (T1) and 3 months post-intervention (T2). Paired $t$-tests and McNemar's test were used to assess changes in outcomes.

Results: A total of 148 men (median age 72) completed the study. There was an increase in OP knowledge $(9.7 \pm 4.3$ to $11.4 \pm 3.3, p<.0001)$ and feelings of susceptibility $(16.5$ \pm 4.3 to $17.4 \pm 4.7, p=.015)$, but a decrease in total selfefficacy $(86.3 \pm 22.9$ to $81.0 \pm 27.6, p=.007)$ from baseline to post-intervention. Men made appropriate changes in their 
overall daily calcium intake ( $p \leq .001)$, and there was uptake of vitamin D supplementation from $44 \%(\mathrm{n}=65)$ to $68 \%(\mathrm{n}=$ 99) $\left(\chi^{2}=24.6, p<.0001\right)$. Men with bone loss (osteopenia or OP) had a greater change in susceptibility $(1.9 \pm 4.3$ vs. -0.22 $\pm 4.2, p=.005)$ compared to men with normal bone density.

Discussion \& Conclusion: Our results provide preliminary evidence that a multi-component intervention such as the one described can lead to increased knowledge, feelings of susceptibility regarding OP, and uptake of some HBBs.

Systematic Review of the Evidence for Trails B Cut-Off Scores in Assessing Fitness-To-Drive

\author{
M. Roy, F. Molnar \\ Department of Geriatric Medicine, University of Ottawa, \\ Ottawa, ON, Canada
}

Background/Purpose: Fitness-to-drive guidelines recommend employing the Trailmaking B test (a.k.a. Trails B), but do not provide guidance regarding cut-off scores. There is ongoing debate regarding the optimal cut-off score on the Trails B test.

Objective: To address this controversy by systematically reviewing the evidence for specific Trails B cut-off scores (e.g., cut-offs in both time to completion and number of errors) with respect to fitness-to-drive.

Methods: Systematic review of all prospective cohort, retrospective cohort, case-control, correlation, and crosssectional studies reporting the ability of the Trails B to predict driving safety that were published in English-language, peer reviewed journals.

Results: 47 articles were reviewed. None of the articles justified sample sizes via formal calculations. Cut-off scores reported based on research include: 90 seconds, 147 seconds, 180 seconds, and $<3$ errors.

Discussion \& Conclusion: There is support for the previously published Trails B cut-offs of 3 minutes or 3 errors (the ' 3 or 3 rule'). Major methodological limitations of this body of research were uncovered including: 1) lack of justification of sample size leaving studies open to Type II error (i.e., false-negative findings), and 2) excessive focus on associations rather than clinically useful cut-off scores.

Utilizing the CanMEDS Framework to Organize and Improve the University of Toronto's Geriatric Medicine Academic Half-Day
N. Varshney ${ }^{1}$, B. Liu ${ }^{1}$, N. Varshney ${ }^{1}$, J. Goguen ${ }^{2}$

${ }^{I}$ Geriatric Medicine, Department of Medicine, University of Toronto, Toronto, ON, and ${ }^{2}$ Endocrinology, Department of Medicine, University of Toronto, Toronto, ON, Canada

Background/Purpose: The Geriatric Medicine (GM) academic half-day (AHD) at the University of Toronto is targeted to structured teaching of the CanMEDS roles. This seminar series must fulfill learners' needs, GM program mandates, and the RCPSC standards for structured education. Given that the University of Toronto has the largest GM program in Canada, the aim is to produce a competency-based AHD framework that can be translated to other Canadian GM programs.

Methods: The RCPSC CanMEDS framework for educational design was utilized. A literature review and a national needs assessment surveying the trainees were conducted. Subsequently, an audit and blueprint of the current AHD curriculum at the University of Toronto were completed. Those domains that were less emphasized were the focus of improvement. Suggestions were made through an educational consultation to improve the structured teaching.

Results: The literature review found no publications related to a Canadian GM AHD curriculum. The needs assessment demonstrated satisfaction in training of all domains, but lesser satisfaction in three areas: the sciences of aging, ethical and legal issues, and formal teaching of the Manager role. The four most formally taught GM specific enabling competencies were Medical Expert 2.1, Manager 1.3, Scholar 3.2, and Medical Expert 3.1. An educational consultation provided practical suggestions for improvement.

Discussion \& Conclusion: The AHD at the University of Toronto is one example of structured teaching, but as a 2-year, weekly seminar series, GM residents invest a great amount of time in this formal education. Peer-reviewed educational tools are available to further enhance the AHD teaching. Improvements to meet the needs of the learner, program, and RCPSC are currently being implemented.

Expanding Care in the Retirement Home Setting: Memory Screening Project and Medchecks-Can We Make a Difference?

G. Lemay ${ }^{1}$, W. Dalziel ${ }^{2}$, S. Bhatti ${ }^{3}$

${ }^{1}$ Internal Medicine/Geriatric Medicine, Ottawa, ON; ${ }^{2} D e-$ partment of Medicine, Division of Geriatrics, Ottawa, ON; ${ }^{3}$ Desjardins Pharmacy, The Ottawa Hospital, Ottawa, ON, Canada

Background/Purpose: Medication-related problems are common, costly, associated with poor outcomes, and are 
potentially preventable in older adults. Older adults with cognitive impairment are at higher risk of adverse drug reactions. The retirement home (RH) setting is a prime opportunity to intervene to screen for cognitive impairment and for medication review.

Methods: This project is a two-phase project taking place in a RH setting. The first phase included resident chart review for diagnosis of dementia or MCI, then cognitive screening using the Dementia Quick Screen (Mini-Cog \& animal fluency). Screen failure lead to full assessment. The second phase included an intervention with Medchecks by pharmacist using the anticholinergic load scale and the Ottawa Top Ten Tool (OTTT). OTTT was developed after a thorough review of the literature/available tools with subsequent geriatrician panel review for the Top 10 higher risk drug classes with practical recommendations. All were sent to the treating physician for review. 3-month follow-up was done to identify physician acceptance of recommendations. Barriers to acceptance will be reviewed.

Results: 75 residents were included in study. Per chart, 45 had normal cognition so were included in the memory screen: 32 (71\%) failed screening. Medchecks were done on 48 residents (16 with dementia). Total of 78 recommendations (range $0-5 \&$ mean 1.6 per resident) were made. 11 $(14 \%)$ anticholinergic-related, 11 (14\%) OTTT-related, and $56(72 \%)$ were other pharmacist recommendations. 31(40\%) recommendations were accepted by treating physician; 4 (5\%) were rejected; 43 (55\%) pending.

Discussion \& Conclusion: Cognitive screen and Medchecks using the new OTTT \& anticholinergic load scale should be incorporated in RH setting to improve care of this aging population.

\section{Dynapenia is Associated with Gait Variability in Com- munity-Dwelling Older Adults}

A. Islam, I. Anton-Rodrigo, K. Gopaul, M. Montero-Odasso Lawson Gait and Brain Laboratory, Geriatric Medicine, Parkwood Hospital, London, ON, Canada

Background/Purpose: The loss of muscle mass, sarcopenia, in older adults is an important marker of frailty due to the association with mobility decline, falls, fractures, and mortality. However, dynapenia, the loss of muscle strength, has been shown to manifest earlier than sarcopenia, and is more consistently associated with disability and mortality. It is unknown whether dynapenia is associated with early gait disturbances, specifically gait variability. Gait variability is a measure of gait regulation, and high gait variability has been proposed as an early marker of mobility decline and a predictor of falls. Therefore, our aim was to determine if dynapenia in community older adults is associated with poorer gait performance, specifically high gait variability.

Methods: In 184 community-dwelling older adults (aged $\geq 75$ ), muscle weakness was assessed by measuring the average grip strength in the dominant hand using a handheld dynamometer. Gait variables were assessed under "usual" and "fast" pace conditions using an electronic walkway. Relative risk analysis evaluated the association of muscle weakness to each of the gait parameters.

Results: Older male adults in the lowest quartile of grip strength $(<20.67 \mathrm{~kg})$ had slower gait velocity [mean $\% \mathrm{CoV}$ $(\mathrm{SD})=82.93(34.51)][\mathrm{RR}(95 \% \mathrm{CI})=1.53(0.58,4.06)]$, and increased stride time variability [mean $\% \mathrm{CoV}(\mathrm{SD})=$ $5.81(1.94)][\mathrm{RR}(95 \% \mathrm{CI})=1.71(0.82,3.57)]$, then those in the highest quartile of grip strength $(\geq 32.33 \mathrm{~kg}$ ). Results were similar in female participants.

Discussion \& Conclusion: Our findings have interesting clinical implications because muscle strength assessments can be used in the clinic as an early screening tool to detect those with high gait instability, risk of falls, and mobility decline.

\section{Using Mobile Health Technologies to Support Healthy Aging at Home}

\section{W. Sun, D. Doran \\ University of Toronto, Toronto, ON, Canada}

Background/Purpose: The purpose of this study is to investigate the integration of two non-intrusive approaches to monitoring home care clients' activity level, along with access to best practice guidelines for clinicians at the point of care. A prototype Remote Activity Monitoring and Guidelines System has been developed that uses a GPS-equipped Blackberry to monitor an elderly client's mobility outside the home. The System includes a pressure-sensitive mat that is placed under a regular bed mattress and can monitor sleep disturbances, and how long it takes to enter and exit the bed.

Methods: A proxy client who is over the age of 65 with chronic health issues was invited to carry a Blackberry and to use a pressure sensitive mat to collect data about the client's physical activity. After a period of 7 days, 4 different nurses made home visits to the proxy client, where a research member observed clinicians interacting with the prototype System in the client's home.

Results: The findings indicated the value of the mobilityrelated data to gerontological clinicians when they plan care 
to address the aging needs of their home care clients. The results also suggested the usefulness and placement of the Best Practice Guidelines in the electronic user interface. The observational data generated information about the clinicians' needs and interaction with the prototype in actual home care setting.

Discussion \& Conclusion: This study provides important implications about the value of remote monitoring technology in providing clinical support to assist gerontological clinicians' decision-making process when planning care for seniors in home care settings.

\section{Evaluating Accessibility Time to Geriatric Medicine Rehabilitation Services}

\author{
X.J. Liu ${ }^{1}$, J.A. Morais ${ }^{2}$ \\ ${ }^{1}$ Faculty of Medicine, McGill University, Pierrefonds, $Q C$, \\ and ${ }^{2}$ Division of Geriatric Medicine, McGill University, \\ Montreal, QC, Canada
}

Background/Purpose: Receiving rehabilitation enables geriatric patients to regain their function prior to return home. However, long waiting times associated with access to rehabilitation are detrimental to the quality of care for geriatric patients.

Methods: Geriatrics consults at the Royal Victoria Hospital and the Montreal General Hospital for 1 year were examined. Relevant information in the consultations was extracted. OACIS was consulted to determine the date of discharge to rehabilitation or home and the number of medications prescribed. The admission/discharge logbooks of the Geriatrics Units were also examined to determine the date of transfer to these units. Statistical analysis was performed on these data using SPSS. A survey of geriatric health professionals determined the reasonable waiting time.

Results: The mean waiting time was 11.4 days for outside rehabilitation or home and 4.3 days for the Geriatrics Units. A theoretical reasonable waiting time of 48 hours was defined from a survey of health professionals. Only $7.5 \%$ of patients were admitted within this theoretical time frame to outside facilities or home and $44 \%$ to a Geriatrics Unit. We didn't find any patient characteristics (age and number of medications) that contributed to explain the waiting time for rehabilitation.

Discussion \& Conclusion: This study documents the long waiting time for patients who were recommended for rehabilitation by the Geriatric Consultation Team. The percentage of patients whose waiting time was lower or equal to the reasonable waiting time set by geriatrics health professionals was quite low. However, the waiting times for the Geriatrics
Units were significantly lower than those for outside facilities or home. Discharging efficiently to rehabilitation could decrease length of stay and improve patient turnover.

\section{Osteoporosis Management and Fracture Prevention in Long-Term Care: a Family Member Preference Survey}

K. Shah ${ }^{1}$, A. Maher ${ }^{2}$, L. Pickard ${ }^{2}$, L. Lohfeld ${ }^{2}$, M-L. van der Horst ${ }^{2}$, C. Skidmore ${ }^{2}$, C. Kennedy ${ }^{2}$, G. Ioannidis ${ }^{2}$, A. Papaioannou $^{2}$

${ }^{1}$ Health Sciences, McMaster University, Hamilton, ON, and ${ }^{2}$ Department of Medicine, McMaster University, Hamilton, ON, Canada

Background/Purpose: Hip fracture is the leading cause of transfer to acute care for long-term care (LTC) residents. Osteoporosis and falls put LTC residents at a high risk for fractures that lead to pain, loss of mobility, heavy costs to patients their families and to the health-care system, and increased mortality. Effective prevention includes pharmaceutical and non-pharmacological interventions to decrease fractures.

Methods: We conducted a pilot telephone survey of LTC residents' family members to inform investigators who are adapting the 2010 Clinical Practice Guidelines for the Diagnosis and Management of Osteoporosis for use in LTC. The 10 questions addressed awareness of and preference for falls and fracture prevention interventions available in LTC. Data were analyzed using frequency counts for closed-ended questions and Thematic Framework Analysis for the openended ones.

Results: $91 \%$ of the 11 respondents supported osteoporosis medication use if indicated, while expressing concerns about potential side effects and polypharmacy issues. All respondents supported Vitamin D supplements without any concerns; $82 \%$ supported calcium + vitamin D use, but worried about swallowing difficulties. Participants felt pain prevention and preserving quality of life were among the most important outcomes for their family members, and extending life was among the least important outcomes.

Discussion \& Conclusion: Results from this pilot survey indicate family members support pharmaceutical interventions, per recommendations in the 2010 Clinical Practice Guidelines for reducing the risk of fractures and falls in LTC residents. Many of them believe pain prevention and quality of life are more important than extending life, which should be considered in guideline development for this population. 
Assessment of Potentially Inappropriate Prescribing in Patients with Dementia in Primary Care Using the 2012 Beers Criteria

\section{A. Martin \\ University of Limerick, Limerick, Ireland}

Background/Purpose: Inappropriate prescribing in the elderly population is associated with adverse drug events and increased hospitalization, ultimately reducing quality of life and increasing mortality rates. The aim was to measure the prevalence of inappropriate prescribing in elderly patients with dementia at Ayrfield Medical Practice in Kilkenny, Ireland. The 2012 Beers Criteria was the standard used for comparison. The Beers Criteria was developed to improve the quality of care for elderly patients and provide physicians with a guideline for safe prescribing.

Methods: Medical charts of 80 patients $\geq 65$ years old with documented dementia at a primary care centre were studied. The range of age was between 65 and 98 years of age, the mean age was 83.5 years ( \pm SD 9.3). Of the patients studied, $67.5 \%$ were female. Patients' current diagnoses and medications were documented and the Beers Criteria was applied to measure the prevalence of inappropriate prescribing.

Results: The mean number of diagnoses per patient was 3.3 ( \pm SD 1.8) and the mean number of medications prescribed per patient was 9.3, ranging from 0-22. Beer's criteria identified a total of 129 inappropriate medications in $72.5 \%$ (58) of patients. Medications with strong anticholinergic properties (antidepressants, antihistamines, antiparkinson agents, skeletal muscle relaxants, antipsychotics, antimuscarinics, and antispasmodics) accounted for the majority of inappropriate medications and were prescribed to $66.3 \%(53)$ of patients.

Discussion \& Conclusion: Potentially inappropriate drug prescribing is widespread among elderly patients. Regular review of medications by a primary care team and cessation of inappropriate medications should be incorporated into intervention strategies to reduce the number of inappropriately prescribed medications and associated adverse outcomes.

Exploring Teaching About Potentially Inappropriate Medications in Geriatrics Patient on CTUs: Are We Preparing our Trainees to Prescribe for Older Patients?

\author{
Y. Hui ${ }^{1}$, L.L. Diachun ${ }^{2}$, L. Lingard ${ }^{3}$, M. Goldszmidt ${ }^{4}$ \\ ${ }^{I}$ The Schulich School of Medicine and Dentistry, London, \\ ON; ${ }^{2}$ Division of Geriatric Medicine, St. Joseph's Health \\ Care, London, ON; ${ }^{3}$ Centre for Education Research \& Inno- \\ vation, London, ON; ${ }^{4}$ Division of General Internal Medicine
}

and Centre for Education Research \& Innovation, London, ON, Canada

Background/Purpose: Medication-related adverse events are a significant cause of morbidity and mortality in the geriatric population. With the percentage of Canadians over age 65 expected to double within the next 20 years, educating medical trainees about appropriate prescribing of medications for geriatric patients is becoming increasingly important. Using the internal medicine teaching ward, this study explored the teaching discussions that occur with respect to prescribing, and the use of potentially inappropriate medications (PIMs).

Methods: Four admission histories for elderly patients were scripted to include learning opportunities regarding geriatric prescribing. A simulated student orally presented 1-3 admission histories to each of 24 internal medicine ward attending physicians (12 geriatricians and 12 internists) who were instructed to respond as they normally would during morning rounds. Semi-structured interviews following the case discussions explored how attending physicians chose the topics they talked about. Transcribed audio-recordings of 66 case review discussions were analyzed using template and inductive analysis for teaching scripts pertaining to PIMs.

Results: Twenty of 24 interviews involving the review of 54 patient cases have been completed. Geriatrician and non-geriatrician attendings varied in terms of their degree of uptake of the geriatric prescribing teaching and learning opportunities built into the cases. Preliminary analysis of 20 completed interviews will be presented.

Discussion \& Conclusion: Teaching about geriatric prescribing and PIMs on the internal medicine clinical teaching wards can play a crucial role for the care of geriatric patients. Our study is the first to examine the teaching discussions around the prescription of medications for older adults in the context of the general medicine ward.

\section{Risk for Frailty in Middle-Aged and Older Americans is Associated with Maternal Age}

T.D. Brothers ${ }^{1}$, O. Theou ${ }^{1}$, M.K. Andrew ${ }^{2}$, T.D. Brothers ${ }^{1}$, K. Rockwood ${ }^{2}$

${ }^{1}$ Geriatric Medicine Research, Dalhousie University, Halifax, NS, and ${ }^{2}$ Department of Medicine, Dalhousie University, Halifax, NS, Canada

Background/Purpose: Children born to mothers of advanced or adolescent ages face increased risk for multiple health problems. In this study, we investigated whether individuals born to mothers of these ages were more likely to experience frailty later in life. 
Methods: This was a retrospective cohort study of the Health and Retirement Survey, including 3,080 Americans age $50+($ mean $=58.2 \pm 5.5$ years, $54 \%$ women) for whom maternal age data were collected. Frailty was assessed using a 33-item frailty index; participants with scores 0.25 were considered frail. Maternal age at participants' time of birth was categorized as older ( 35 years) or younger ( $<20$ years), compared to a reference group (20-34 years).

Results: Mean maternal age was $22.7 \pm 6.5$ years. After controlling for participants' age, gender, and education level, being born to an older mother was associated with higher risk for frailty compared to the reference group $(\mathrm{OR}=1.61$, $95 \% \mathrm{CI}=1.05-2.48$ ), as was being born to a younger mother $(\mathrm{OR}=1.40,95 \% \mathrm{CI}=1.15-1.71)$. When maternal education level was added to the regression model, being born to an older mother was no longer associated with higher risk for frailty $(\mathrm{OR}=1.52,95 \% \mathrm{CI}=0.98-2.34)$, but being born to a younger mother remained associated with higher risk, at a similar level $(\mathrm{OR}=1.41,95 \% \mathrm{CI}=1.15-1.72)$.

Discussion \& Conclusion: Among middle-aged and older Americans, being born to an older mother is not associated with greater risk for frailty once maternal education is taken into account. However, being born to an adolescent mother is associated with higher risk for frailty later in life, regardless of maternal education level.

Social Vulnerability is an Independent Predictor of Mortality in Community-Dwelling Middle-Aged and Older Europeans

L. Wallace, O. Theou, M. Andrew, K. Rockwood

Department of Medicine, Dalhousie University, Halifax, NS, Canada
Background/Purpose: Social vulnerability has been shown to be associated with mortality in Canadian populations. The purpose of this study was to investigate whether social vulnerability can predict mortality in middle-aged and older Europeans, after considering frailty.

Methods: This was a secondary analysis of the first wave of SHARE (Survey of Health and Retirement in Europe), which began in 2004 and included a probability-based sample of non-institutionalized participants aged 50+ from 11 European countries. We used the deficit accumulation approach to create a frailty index and a social vulnerability index. The frailty index included 70 health deficits from the physical health, behavioural risks, cognitive function, and mental health sections of the main questionnaire. The social vulnerability index included 29 social factors from the drop-off questionnaire. For each index, an individual's score reflects the proportion of deficits present out of the total possible deficits.

Results: 18,289 participants were included in the analysis (age $65.0 \pm 9.67,45.9 \%$ male). The mean frailty index score was $0.15 \pm 0.11$ and the mean social vulnerability index score was $0.32 \pm 0.09$. Social vulnerability significantly predicted 5 -year mortality when controlling for age and sex (adjusted hazard ratio $=1.33$, confidence interval $1.25-1.42, p<.001)$. This association remained significant when additionally controlling for frailty (adj. HR $=1.09$, CI 1.01-1.17, $p=.02$ ).

Discussion \& Conclusion: Similarly to Canadian populations, social vulnerability appears to be an important component for mortality risk stratification in middle-aged and older Europeans. Future investigations are needed to focus on the clinical implications of social vulnerability in older patients.

\section{NON-TRAINEE POSTER ABSTRACTS}

\section{The Impact of Different Types of Exercise on Arterial Stiffness in Older Adults at Very High Cardiometabolic Risk}

K. Madden, C. Lockhart, D. Cuff, G. Meneilly University of British Columbia, Vancouver, BC, Canada

Background/Purpose: The relationship between increased arterial stiffness and cardiovascular mortality is well-established in older adults. Short-term vigorous exercise interventions have been shown to reduce arterial stiffness in older adults with T2DM. We examined whether training type (aerobic training versus strength training) influences the improvement in arterial compliance in older adults with Type 2 diabetes complicated by co-morbid hypertension and hyperlipidemia.

Methods: A total of 45 older adults (mean age $72.3 \pm 0.7$ years) with diet-controlled or oral hypoglycemic-controlled T2DM, hypertension, and hypercholesterolemia were recruited. Subjects were randomly assigned to one of three groups: an aerobic group (6 months vigorous aerobic exercise, AT group, $\mathrm{n}=20$ ), a strength training group (6 months strength training, ST group, $\mathrm{n}=15$ ), and a control group (no training, $C$ group, $n=10)$. Exercise sessions were supervised by a certified exercise trainer three times per week. Arterial stiffness was measured as pulse-wave velocity (PWV) using the Complior device. 
Results: There was a significant difference in the response to training (group $\times$ time) between the AT and NA groups for both radial $(p=.011)$ and femoral $(p=.017)$ PWV. This was primarily due to an improvement in the AT group after 3 months training as compared to control ( $p<.001$ radial PWV; $p<.001$ femoral PWV), that was not maintained at the 6-month mark for either radial or femoral PWV.

Discussion \& Conclusion: Our findings indicate that in older adults with T2DM, long-term strength training resulted in no improvement in measures of arterial stiffness, while aerobic exercise resulted in short-term improvements in arterial stiffness that became attenuated over the long term.

Care of the Elderly Program: Training Future Physicians and Promoting Research and Clinical Excellence for the Medically Complex Senior

L. Charles ${ }^{1}$, J. Triscott ${ }^{2}$, B. Dobbs ${ }^{2}$, R. McKay ${ }^{1}$

${ }^{I}$ Division of Care of the Elderly, Department of Family Medicine, University of Alberta, Edmonton, $A B$, and ${ }^{2}$ University of Alberta, Edmonton, AB, Canada

Background/Purpose: Our program aims to provide physicians with Enhanced Skills in Care of the Elderly training. The program has undergone significant educational changes in the last year.

Methods: The COE Program was established at the University of Alberta in 1993. To date, 51 residents have completed the program. Program description: 6 months to 1 year Enhanced Skills Diploma Program with core program requirements including geriatric inpatient, geriatric psychiatry, ambulatory, continuing care, and outreach. There is a longitudinal clinic component and a research project requirement. The program is designed to cover 85 core competencies encompassing the CanMEDS-Family Medicine Roles.

Results: With the increased complexity of the frail elderly we are expanding the program to a 1-year program for the majority of residents, with an exit exam upon completion. This exit exam is comprised of MCQ and geriatric assessment observation with patient encounter. We have been able to increase our positions to four 1-year positions from four 6-month positions. With the increase of the program to 1 year, we have added new rotations in Palliative Care and significantly developed the community experience with rotations in Continuing Care which includes Supportive Living and a Home Living rotation. We have also introduced the electronic Competency Based Achievement System to give formative feedback to our residents.

Discussion \& Conclusion: There is a need for Care of the Elderly physicians to provide clinical care, as well as educational, administrative, and research roles to meet the health-care needs of medically complex seniors. We have made changes to our program to prepare residents for these roles.

\section{A Post-Hoc Analysis of a Randomized, Double-Blinded, Placebo-Controlled Trial of Gabapentin on Post-Opera- tive Delirium Following Total Knee Arthroplasty}

C. Wong ${ }^{1}$, K. Dighe ${ }^{2}$, H. Clarke ${ }^{3}$, C. McCartney ${ }^{4}$

${ }^{1}$ St. Michael's Hospital, Toronto, ON; ${ }^{2}$ York Central Hospital, Richmond Hill, ON; ${ }^{3}$ UHN Toronto General Hospital, Toronto, ON; ${ }^{4}$ Sunnybrook Health Sciences Centre, Toronto, ON, Canada

Background/Purpose: Post-operative delirium is associated with pain but also from the use of analgesics. Gabapentin has an opioid sparing effect and reduces pain in the acute post-operative period. The study objective was to determine the treatment effect of perioperative gabapentin on the incidence of post-operative delirium among elective total knee arthroplasty (TKA) patients.

Methods: 161 patients with American Society of Anesthesiology (ASA) physical status class I-III scheduled for elective total knee arthroplasty at an orthopedic centre were randomized to receive gabapentin $200 \mathrm{mg}(\mathrm{n}=83)$ or placebo $(\mathrm{n}=78)$ before surgery and up to 3 days post-operatively. Incident delirium in the post-operative period was determined by a validated chart abstraction tool. A subset of charts was abstracted by two independent reviewers to determine inter-rater reliability. Data abstractors and patients were blinded to the study drug allocation.

Results: Inter-rater agreement was good $(\kappa=0.83)$. Baseline characteristics, co-morbidities, type of anesthesia and analgesia, and previous exposure to gabapentin between the 2 groups were similar. Incident delirium in gabapentin (12\%) and placebo $(9 \%)$ groups was not significantly different ( $p=.53$; absolute risk reduction $-3.1 \%, 95 \%$ CI -12.5 to $6.4 \%$ ). The mean duration of delirium in both groups was 1 day.

Discussion \& Conclusion: Perioperative gabapentin was not effective for the prevention of post-operative delirium in elective TKA patients nor did gabapentin have an effect on delirium duration.

\section{Multimorbidity, Disability, and Mortality}

P. St John ${ }^{1}$, V. Menec ${ }^{2}$, S. Tyas ${ }^{3}$, R. Tate ${ }^{1}$

${ }^{1}$ University of Manitoba, Winnipeg, MB, Canada $;{ }^{2}$ Centre on Aging, University of Manitoba, Winnipeg, $M B$, and ${ }^{3}$ University of Waterloo, Waterloo, ON, Canada 
Background/Purpose: The objectives are: to describe factors associated with multi-morbidity in community-dwelling older adults; and to determine if a simple measure of multimorbidity predicts death over 5 years.

Methods: Analysis of an existing population-based cohort study. Population: 1751 community-dwelling adults, aged 65+, were interviewed and followed over 5 years. Measures: Age, gender, marital status, living arrangement, and education were all self-reported; the Mini-Mental Status Examination (MMSE), the Center for Epidemiologic Studies-Depression (CES-D), and the Older Americans Resource Survey (OARS). The measure of multi-morbidity was a simple list of common health complaints and diseases, followed by an open-ended question of other problems. These were summed and the score ranged from 0 to 16 . Death and time of death were determined over the 5-year interval by death certificate, administrative data, and proxy report. Analysis: Cox proportional hazards models were constructed for time to death.

Results: Multi-morbidity was more prevalent in women, older age groups, those with lower education levels, lower MMSE scores, more depressive symptoms, and higher levels of disability. Multi-morbidity was a strong predictor of mortality in unadjusted models - the Hazard Ratio (HR) and 95\% confidence interval $(95 \% \mathrm{CI})$ was $1.09(1.05,1.12)$. In models adjusting for age, gender, education, marital status, living arrangement, the CES-D, and the MMSE, this effect persisted: the HR and $95 \%$ CI was $1.04(1.00,1.08)$. However, after adjusting for functional status, the effect of multi-morbidity was no longer significant.

Discussion \& Conclusion: Multi-morbidity strongly predicts 5 -year mortality, and the effect may be mediated by disability. The cumulative effect of health problems, however minor, is associated with poor outcomes. Guidelines and clinical care models must consider multi-morbidity.

Is Mobility a Predictor of Complications Post-PCI in Cardiac Patients Over the Age of 85?

\author{
J. Basran ${ }^{1}$, S. Sra ${ }^{2}$, R. Basran 3 \\ ${ }^{I}$ Division of Geriatrics, University of Saskatchewan, Saska- \\ toon, SK; ${ }^{2}$ Department of Medicine, University of Saskatch- \\ ewan, Saskatoon, SK; ${ }^{3}$ Division of Cardiology, University of \\ Saskatchewan, Saskatoon, SK, Canada
}

Background/Purpose: Older patients often pose a challenge to physicians who must determine which patients are good candidates for invasive cardiac procedures, a decision often left to clinical gestalt. The concept of frailty, a multidimensional approach to stratify older patients by physiology and function rather than age, has been associated with poor outcomes. However, due to the lack of consensus on significant measures and the increased time and personnel required, routine frailty assessments are not often used.

Methods: A retrospective chart review was completed on 171 consecutive patients over the age of 85 who underwent PCI between 2007 and 2010. Four outcomes were evaluated: major adverse cardiac event, in-hospital death, increase in creatinine by $>25 \%$, or any in-hospital complication. Sixteen demographic, clinical and frailty variables were studied.

Results: The univariate analysis, using chi square for categorical and $t$-test for continuous variables, found that patients presenting with cardiogenic shock or urgent PCI had an increased risk for each of the four outcomes. A logistic regression with the outcome "any in-hospital complication", found that the "inability to walk without an aid or assistance" (OR 3.9 (95\% CI 1.8, 8.5)) was associated with in-hospital complications.

Discussion \& Conclusion: Our study found that patients over the age of 85 , who were unable to walk without an aid or assistance, were 3.9 times more likely to have a post-PCI in-hospital complication. Asking a patient this simple question about their mobility is both quick and straightforward. A larger prospective study will need to assess whether this type of question could be used as a bedside screening tool to predict poor outcomes in older adults undergoing PCI.

\section{Multimorbidity at a Rehabilitation Hospital: Analysis and Implications}

J. Triscott ${ }^{1}$, D. Campbell-Scherer ${ }^{1}$, R. Hagtvedt ${ }^{2}$, M. Gojmerac ${ }^{3}$ ${ }^{1}$ Department of Family Medicine, University of Alberta, Edmonton, AB; ${ }^{2}$ Department of Business, University of Alberta, Edmonton, $A B ;{ }^{3}$ Faculty of Medicine and Dentistry, University of Alberta, Edmonton, AB, Canada

Background/Purpose: There is paucity of information concerning the epidemiology of multimorbidity in the frail elderly in Alberta. Four rehabilitation wards at a Rehabilitation Hospital have collected data from 2003-2012 for each admission. The de-identified data include ICD-10 diagnosis codes, length of stay (LOS), admission and discharge dates, admission and discharge Functional Independence Measure (FIM) scores, and age of patients. The objective is to begin analyzing and characterizing multi-morbidity in the geriatric population of Alberta.

Methods: Data for 2010-12 were separated. A list of all present ICD-10 codes was formed. ICD-10 codes were put into diagnosis groups, which were then counted. The number of ICD-10 codes per patient was counted. The rate of FIM change (FIM efficiency) was calculated according to 
the equation: (Discharge FIM-Admission FIM)/Length of Stay). Regression analysis was performed to compare the relatedness between FIM Efficiency and Admit FIM, Length of Stay, and Number of Diagnosis Codes.

Results: Initial analysis of codes of interest showed that $0 \%$ of this geriatric population had a code for chronic obstructive pulmonary disease, congestive heart failure, or urinary tract infection. Regression analysis revealed that Admission FIM and LOS are significant with FIM Efficiency, but Number of Diagnosis Codes is not.

Discussion \& Conclusion: The ICD-10 codes do not reflect expected prevalence for major chronic diseases. This may be a result of codes forming a present problem/treatment list, rather than a list of all diagnoses. There is a need for another study to fully describe the epidemiology of multi-morbidity in this population.

\section{What Is the Cost-Effectiveness of Wound Care Interven- tions?}

E. Cogo, J. Antony, G. Sanmugalingham, P.A. Khan, S.E. Straus, A.C. Tricco

Li Ka Shing Knowledge Institute of St. Michael's Hospital, Knowledge Translation Program, Toronto, ON, Canada

Background/Purpose: Wounds, such as diabetic, venous ulcers, pressure ulcers, and surgical wounds, present a significant economic burden on health-care systems. High-quality cost-effectiveness evidence may play a role in considering resource allocation. We conducted a systematic review of cost-effectiveness analyses (CEAs) of wound care interventions to evaluate the methodological quality and cost-effectiveness of this evidence-base.

Methods: Potentially relevant material was identified through searching MEDLINE, EMBASE and the Cochrane Library. Inclusion criteria included CEAs assessing any type of intervention for treating wounds in adults. Two reviewers independently screened search results and abstracted data from relevant articles in duplicate. The methodological quality of the included CEAs was appraised using the Drummond tool.

Results: 6199 titles and abstracts and 421 full-text articles were screened for inclusion. Of these, 35 CEAs (including 12 cost-utility analyses) were included. The majority of the included CEAs (69\%) focused on elderly patients. Only 12 CEAs were deemed to be high-quality (including one from Canada). Seven high-quality CEAs found the following interventions were cost-effective: pentoxifylline plus usual care versus standard compression with external treatment, 4-layer high-compression bandages versus short-stretch high-compression bandages, multi-disciplinary community wound care teams versus usual nursing care, hyperbaric oxygen therapy plus standard care versus standard care alone, becaplermin gel containing recombinant human plateletderived growth factor plus standard care versus usual wound care alone, and ertapenem versus piperacillin/tazobactam.

Discussion \& Conclusion: We identified a large research gap in CEAs of wound care interventions, and the quality of the evidence is limited.

\section{Effectiveness of Interprofessional Education on Profes- sional Practice and Health Outcomes}

V. Chau ${ }^{1}$, J. Lee ${ }^{1}$, J. Alston ${ }^{2}$, H. McLeod ${ }^{3}$, K. Tzanetos ${ }^{2}$, M. Zwarenstein ${ }^{4}$, S. Straus ${ }^{5}$

${ }^{1}$ Department of Medicine, Hamilton, ON; ${ }^{2}$ Department of Medicine, Toronto, ON; ${ }^{3}$ Department of Medicine, Division of Geriatric Medicine, Hamilton, ON; ${ }^{4}$ Sunnybrook Health Sciences Centre, North York, ON; ${ }^{5} \mathrm{Li}$ Ka Shing Knowledge Institute, Toronto, ON, Canada

Background/Purpose: Older adults have multiple chronic health and social conditions, requiring expertise from different health-care professionals. With the proportion of older adults increasing, it's important for these professionals to work together effectively. Interprofessional education (IPE) (when two or more professionals learn with, from, and about each other to improve collaboration and quality of care) has been incorporated into policy, systems, and curricula globally. However, the impact of IPE remains unclear. An updated systematic review was performed to assess the effectiveness of IPE interventions on professional practice and health outcomes.

Methods: We searched MEDLINE, CINAHL, and the Cochrane EPOC Register from 2007 to 2010. Additional articles were identified through reference lists and discussion with experts. Randomised controlled trials (RCTs), controlled before and after (CBA), and interrupted time series (ITS) studies of IPE interventions reporting objectively measured or self-reported (validated instrument) patient and/or health-care process outcomes were included. Two reviewers independently assessed potential study eligibility, performed data abstraction, and quality assessments.

Results: Three studies met inclusion criteria. The CBA study reported improvements in presurgical procedure briefings and teamwork behaviours in the operating room of a community hospital. One RCT showed mixed results with no change in adverse patient outcomes, but a reduction in process outcomes (time from decision to perform a 
caesarean section to incision) in a labour and delivery team. Another RCT did not demonstrate an impact on primary care management of asthma.

Discussion \& Conclusion: Although studies suggest some positive impact, the difficulty of drawing conclusions about the effectiveness of IPE remains. Due to the heterogeneous and small number of studies with methodological limitations, further rigorous study designs are warranted.

Associations Between Cognitive Performance and SelfReported Driving Comfort, Abilities, and Behaviours in a Large Cohort of Older Drivers

G. Naglie ${ }^{1}$, M. Rapoport ${ }^{2}$, K. Weegar ${ }^{3}$, D. Cameron ${ }^{4}$, A. Myers $^{5}$, H. Tuokko ${ }^{6}$, N. Korner-Bitensky ${ }^{7}$, S. Marshall ${ }^{3}$, M. Man-Son-Hing ${ }^{3}$, A. Crizzle ${ }^{8}$, The Candrive Research Team (c/o Dr. Shawn Marshall) ${ }^{3}$

${ }^{1}$ Baycrest Geriatric Health Care Centre, Toronto, ON; ${ }^{2}$ University of Toronto, Toronto, ON; ${ }^{3}$ University of Ottawa, Ottawa, ON; ${ }^{4}$ York University, Toronto, ON; ${ }^{5}$ University of Waterloo, Waterloo, ON; ${ }^{6}$ University of Victoria, Victoria, $B C ;{ }^{7}$ Mc Gill University, Montreal, QC; ${ }^{8}$ McMaster University, Hamilton, ON, Canada

Background/Purpose: Cognitive impairment can affect driving performance among older drivers. The objective of this study was to examine the association between selected cognitive measures and self-reported driving comfort, abilities, and behaviours.

Methods: We conducted a cross-sectional analysis of data from the first year of the Candrive II prospective cohort study, a 5-year longitudinal study of healthy drivers aged $70+$ from seven Canadian cities. Cognitive assessment tools included: the Montreal Cognitive Assessment (MoCA) and Trail Making Tests, parts A and B. Driver perceptions were assessed using the Day and Night Driving Comfort scales and the Perceived Driving Abilities scale, while driving practices were captured by the Situational Driving Frequency and Avoidance scales and the Driving Habits and Intentions Questionnaire.

Results: A total of 928 drivers, $62.2 \%$ male, with a mean age of $76.2 \pm 4.8$, were recruited. Univariate regression analyses revealed that the times to complete Trails A and B were modestly associated with self-reported driving avoidance, day and night driving comfort, and perceived driving abilities $(p<.05)$. The association persisted after adjusting for age and sex, as well as variables pertaining to health, vision, mood, and physical functioning. Neither MoCA total score nor errors on Trails A and B were associated with any of the self-reported driving variables $(p>.05)$.
Discussion \& Conclusion: Time to complete Trails A and B was statistically significant, but only modest predictors of self-reported driving comfort, abilities, and behaviours in this cross-sectional analysis. Results from the prospective follow-up of this cohort of older drivers will help clarify the relationship between cognitive performance and self-reported driver perceptions and driving restrictions.

\section{Adapting Diet According to Age in an Adult Acute Care Hospital}

A. Dupras 1 , M. Khaddag 2 , L. Belley², A. Younanian ${ }^{3}$, G. Proulx $^{4}$, R. Monette ${ }^{2}$, S. Lafrenière ${ }^{5}$

${ }^{I}$ Centre Hospitalier Universitaire de Montreal, Département de médecine, Service de gériatrie, Montreal, QC; ${ }^{2}$ Centre Hospitalier Universitaire de Montréal, Service de nutrition clinique, Montreal, QC; ${ }^{3}$ Centre Hospitalier Universitaire de Montréal, Service de gestion des menus, Montreal, QC; ${ }^{4}$ Centre Hospitalier Universitaire de Montreal, Montreal, QC; ${ }^{5}$ Centre-Hospitalier Universitaire de Montréal, Direction des soins infirmiers, Montreal, QC, Canada

Background/Purpose: Hospital malnutrition is prevalent in elderly and contributes to loss of functional status, increases morbidity, mortality, length of stay and cost of care. Nutritional interventions improves outcomes in hospitalized elderly. Systematic service of diet based solely on age is not customary in adult acute care settings.

Methods: As part of the OPTIMAH approach of care, we analyzed the protein and caloric content of every available diet at the Montreal University Hospital and compared it to metabolic requirements of hospitalized elders ( $75 \mathrm{yrs}$ ).

Results: Most diets did not meet increased metabolic needs of the hospitalized elder population. Thus, we elaborated a menu that fulfills nutritional requirements and preferences of elders. New enrichment processes were developed to minimize cost. We modified the distribution process to ensure automatic serving of the OPTIMAH diet to this population. Nutritionists, diet technicians, and nurses on ward received a short training to inform them of the new diet and process of distribution. Nurses and physicians were sensitized to avoid prescribing restricted diets unless part of immediate essential medical treatment. Six months after the availability of the OPTIMAH diet throughout the 3 sites of the Montreal University Hospital, $74 \%$ of elder hospitalized patients were receiving this adapted diet.

Discussion \& Conclusion: The new OPTIMAH diet more closely fulfills the metabolic needs of elders in acute care. It is the first step to prevent in-hospital malnutrition. Adult acute care services should, like pediatric health services, 
offer adapted diets according to age. Government norms and correspondent financing should be readjusted to meet elders' metabolic requirements to prevent costly complications related to hospital-acquired malnutrition.

The Dementia Trilogy: Development of Three Original Booklets About Dementia for Patients and Their Families

\section{E. Rhynold, C. Hobbs, K. Hurley, S. Dougan \\ Horizon Health Network, Saint John, NB, Canada}

Background/Purpose: Education is an important component of dementia treatment for patients and their support networks. To compliment recommending education available from the Alzheimer Society of Canada, practical booklets were developed to improve the written educational material available regarding dementia.

Methods: Hobbs, Hurley and Rhynold wrote three booklets: An Introduction to Dementia, The Dementia Compass, and Later in the Dementia Journey. Dougan designed an eye-catching theme to appeal to a wide audience. These booklets have been piloted in the Horizon Health Network, New Brunswick. A satisfaction survey was given to 25 patients and/or caregivers attending the Geriatric Medicine Ambulatory clinics at St. Joseph's Hospital in Saint John, NB at follow-up appointments. Email feedback was also solicited.

Results: As of September 2012, six sites in New Brunswick have ordered a total of 4151 booklets. Survey results averaged $>9 / 10$ on the visual analog scale with a score of 10 indicating "very helpful". Email feedback was positive with some suggestions for improvements. By survey, clinic attendees often indicated they were not interested in reading the material online.

Discussion \& Conclusion: Future directions: The writing team has always made the free distribution of these booklets their priority. Now that these booklets are available, the focus has shifted to increase distribution through written and online versions.

\section{Fracture Risk Assessment in Long-Term Care}

M. Wall ${ }^{1}$, L. Lohfeld ${ }^{2}$, A. Papaioannou ${ }^{3}$, A. Moser $^{4}$, L. Giangregorio $^{5}$, G. Ioannidis ${ }^{3}$, C. Kennedy ${ }^{3}$, S. Morin ${ }^{6}$

${ }^{1}$ Mc Gill University Health Centre, Research Institute, Montreal, QC; ${ }^{2}$ Clinical Epidemiology \& Biostatistics, McMaster University, Hamilton, ON; ${ }^{3}$ Department of Medicine, McMaster University, Hamilton, ON; ${ }^{4}$ Department of Family and Community Medicine, University of Toronto, Toronto,
ON; ${ }^{5}$ Department of Kinesiology, University of Waterloo, Waterloo, ON; ${ }^{6}$ Department of Medicine, McGill University, Montreal, QC, Canada

Background/Purpose: Osteoporosis Canada's Clinical Practice Guidelines for the Diagnosis and Management of Osteoporosis $(\mathrm{OCG})$ provide guidance for the management of individuals 50 years and older at risk for fractures. However, OCG cannot benefit long-term care (LTC) residents if physicians perceive barriers to their application. Our objectives are to explore current practices to fracture risk assessment by LTC physicians, and describe barriers to applying OCG for fracture assessment and prevention in LTC.

Methods: A cross-sectional survey was conducted with the Ontario Long-Term Care Physicians Association using an online questionnaire. Closed-ended responses were analyzed using descriptive statistics and thematic framework analysis for open-ended responses.

Results: We contacted 347 LTC physicians; 88 submitted completed surveys $(81 \%$ men, mean age 60 years (SD 11), average 32 [SD 11] years in practice). $87 \%$ of LTC physicians considered the prevention of fragility fractures important, but a minority (34\%) reported using validated fracture risk assessment tools, while $33 \%$ did not use any. Clinical risk factors recommended by OCG for assessing fracture risk considered applicable included: glucocorticoid use $(99 \%)$, fall history (93\%), age (92\%), and fracture history $(90 \%)$. Recommended clinical measurements considered applicable included: weight $(84 \%)$, TSH (78\%), creatinine (73\%), height (61\%), and GetUp-and-Go (60\%). Perceived barriers to assessing fracture risk included difficulty acquiring necessary information (35\%) due to lack of access to tests (bone mineral density, X-rays) or obtaining medical history; resource constraints (30\%); and a sentiment that assessing fracture risk is futile in this population because of short life expectancy and polypharmacy (28\%).

Discussion \& Conclusion: These findings highlight the necessity to adapt the OC guidelines so they are evidencebased and applicable to LTC, and to disseminate them to LTC physicians.

\section{Outcomes Utilized to Assess Alzheimer's Disease from a} Systematic Review and Survey of Decision-Makers

C. Soobiah, S.E. Straus, E. Blondal, H. Ashoor, M. Ghassemi, P.A. Khan, J. Ho, S. Berliner, A.C. Tricco

Li Ka Shing Knowledge Institute at St. Michael's Hospital, Toronto, ON, Canada

Background/Purpose: We conducted a systematic review examining the comparative safety and efficacy of cognitive 
enhancers for patients with Alzheimer's disease (AD). Numerous outcomes to assess AD were identified but selecting optimal ones for inclusion in our systematic review remained unclear. We compiled the identified outcomes and surveyed decision-makers to identify relevant outcome measures for inclusion in our systematic review.

Methods: A systematic review was conducted on cognitive enhancers for AD by searching MEDLINE, EMBASE, and the Cochrane Library. Subsequently, two reviewers independently abstracted outcome measures used to assess cognition, function, behaviour, and global status. The identified outcome measures were compiled and sent to 36 clinicians (geriatricians from the Divisions of Geriatric Medicine at the University of Toronto and McMaster University) and 17 health policy-makers (from Health Canada) using FluidSurvey. Participants voted on the utility of 72 cognition measures, 29 function measures, 13 behavioural measures, and 12 global status measures using a 7-point Likert scale ranging from not important to most important. The scores for each scale were averaged to obtain a rating per scale.

Results: $\quad 60 \%$ of invitees completed the survey. The average ratings per scale ranged from 6.50 to 2.97 . The top-rated scale for cognition was the Trail Making test (average score 5.80), for function was the Activities of Daily Living (6.50), for behaviour was the Brief Neuropsychiatric Inventory (5.53), and for global status was the Clinician Interview-based Impressions of Change plus Caregiver Input (6.10). These results were used to inform data abstraction for our systematic review.

Discussion \& Conclusion: Our results can inform clinicians and researchers about relevant outcomes to assess patients with AD.

Cognitive Enhancers for Alzheimer's Disease: a Systematic Review and Network Meta-Analysis

C. Soobiah ${ }^{1}$, A.C. Tricco ${ }^{1}$, S. Berliner ${ }^{1}$, J. Ho ${ }^{1}$, C. $\mathrm{Ng}^{1}$, M.H. Chen $^{1}$, B. Hemmelgarn ${ }^{2}$, S. Majumdar ${ }^{3}$, S.E. Straus ${ }^{1}$

${ }^{1}$ Li Ka Shing Knowledge Institute at St. Michael's Hospital, Toronto, ON; ${ }^{2}$ Department of Medicine and Community Health Sciences, University of Calgary, Calgary, AB; ${ }^{3} \mathrm{Di}$ vision of General Internal Medicine, University of Alberta, Edmonton, AB, Canada

Background/Purpose: In Canada, cognitive enhancers such as donepezil, rivastigmine, galantamine, and memantine have been approved for use in AD. Our objective was to examine the comparative efficacy and safety of these agents through network meta-analysis (NMA). NMA is an extension of traditional meta-analysis, and covers both indirect treatment comparison and mixed treatment comparison.
Methods: Experimental and observational studies were identified through searching electronic databases (e.g., MEDLINE, AgeLine) from inception to 2011. Studies reporting on adverse events, cognition (e.g., Alzheimer's disease assessment scale - cognitive subscale [ADAS-cog]), function, behaviour or global status were included. Reviewers independently screened search results and abstracted data from relevant articles in duplicate. Methodological quality was appraised using the Cochrane Risk of Bias for experimental studies and the Newcastle Ottawa scale for observational studies. Random effects and network metaanalyses were conducted.

Results: A total of 132 randomized controlled trials, 4 nonrandomized clinical trials, 2 controlled before-after studies, and 44 cohort studies were included after screening 15,676 titles and abstracts and 964 full-text articles. Preliminary results from the NMA indicate the following drugs performed better than others on cognition as per the ADAS-cog scale (listed in descending order): donepezil $10 \mathrm{mg}$, donepezil $5 \mathrm{mg}$, galantamine $16-24 \mathrm{mg}$, and memantine $20 \mathrm{mg}$. For nausea, use of the following drugs resulted in lower proportions of patients experiencing nausea (listed in descending order): memantine $20 \mathrm{mg}$, rivastigmine patch $9.5 \mathrm{mg}$, placebo, and donepezil $5 \mathrm{mg}$.

Discussion \& Conclusion: Donepezil 5-10 mg was most effective at improving cognition for patients with AD. The cognitive enhancer with the lowest risk of nausea was memantine $20 \mathrm{mg}$. These results can be used by patients and clinicians to tailor their AD treatment by specific cognitive enhancers.

\section{Cognitive Enhancers for Mild Cognitive Impairment: a} Systematic Review and Meta-Analysis

C. Soobiah, S. Berliner, J. Ho, C. Ng, B. Dong, S.E. Straus, A.C. Tricco

Li Ka Shing Knowledge Institute at St. Michael's Hospital, Toronto, ON, Canada

Background/Purpose: Individuals with mild cognitive impairment (MCI) suffer from memory problems without significant limitations in activities of daily living (ADL). Cognitive enhancers are used to treat dementia, but their effectiveness for MCI is unclear. We conducted a systematic review to examine the comparative efficacy and safety of cognitive enhancers for patients with MCI.

Methods: Experimental studies were identified through searching electronic databases (e.g., MEDLINE, EMBASE). Studies examining cognitive enhancers in MCI and reporting on adverse events, cognition (Mini-Mental State Exam 
[MMSE], Alzheimer's disease assessment scale - cognitive subscale [ADAS-cog]) or function (Alzheimer's disease cooperative study: ADL inventory [ADCS-ADL]) were included. Two reviewers independently screened search results, abstracted data, and appraised risk of bias using the Cochrane risk of bias tool. Random effects meta-analysis was conducted.

Results: Nine randomized controlled trials were included after screening 15,676 titles and abstracts and 964 full-text articles. No significant findings were observed for impact on cognition (MMSE: 3 RCTs, mean difference [MD] 0.14, $95 \%$ confidence interval [CI] $-0.22,0.50$, ADAS- $\operatorname{cog} 5$ RCTs, MD - $0.52,95 \%$ CI $-1.09,0.06$ ), although there was a trend towards favouring cognitive enhancers. Similarly, function was not significantly impacted (ADCS-ADL, 2 RCTs, MD $0.30,95 \%$ CI $-0.26,0.86)$ and no trend was observed. Cognitive enhancers were associated with a higher risk of nausea (5 RCTs, relative risk [RR] 2.95, 95\% CI 2.48, 3.52), diarrhea (5 RCTs, RR 2.71, 95\% CI 1.90, 3.85), and vomiting (3 RCTs, RR $4.40,95 \%$ CI $3.21,6.03)$.

Discussion \& Conclusion: Cognitive enhancers did not improve cognition or function among patients with $\mathrm{MCI}$ and were associated with a greater risk of nausea, diarrhea, and vomiting.

\section{Adverse Cardiac Events in Older Patients Receiving Venlafaxine: a Population-Based Study}

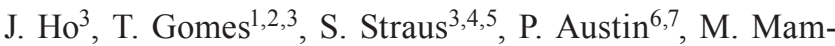
dani $^{2,3,6,7}$, D. Juurlink $\mathrm{k}^{6,7,8,9}$

${ }^{I}$ The Institute for Clinical Evaluative Sciences, Toronto, ON; ${ }^{2}$ The Leslie Dan Faculty of Pharmacy, University of Toronto, Toronto, ON; ${ }^{3} \mathrm{Li} \mathrm{Ka}$ Shing Knowledge Institute, St. Michael's Hospital, Toronto, ON; ${ }^{4}$ Institute of Health Policy, Management and Evaluation, University of Toronto, Toronto, ON; ${ }^{5}$ Division of Geriatric Medicine, Department of Medicine, University of Toronto, Toronto, ON; ${ }^{6}$ The Institute for Clinical Evaluative Sciences, Toronto, ON; ${ }^{7}$ Institute of Health Policy, Management and Evaluation, University of Toronto, Toronto, ON; ${ }^{8}$ Department of Medicine, University of Toronto, Toronto, ON; ${ }^{9}$ The Sunnybrook Research Institute, Toronto, ON, Canada

Background/Purpose: Venlafaxine is a commonly prescribed antidepressant, but it is unknown whether its noradrenergic effects impart an increased cardiovascular risk.

Objective: To examine the cardiac safety of venlafaxine relative to sertraline in older patients.

Methods: We conducted a retrospective cohort study using administrative health-care databases in Ontario, Canada. We included all patients aged 66 years or older who commenced treatment with either venlafaxine or sertraline between April 1, 2000 and March 31, 2009. We used inverse probability of treatment weighting (IPTW) with the propensity score to account for observed systematic differences between the two treatment groups. The primary outcome was a composite of death or hospitalization for acute myocardial infarction or congestive heart failure within the first year of therapy. In secondary analyses, each outcome was examined separately.

Results: We studied 48,876 patients initiated on venlafaxine and 41,238 patients initiated on sertraline. Of these, 4259 $(8.7 \%)$ and 3459 (8.4\%) experienced the primary outcome, respectively. We found no significant difference in the risk of adverse cardiac events with venlafaxine relative to sertraline (hazard ratio 0.97 ; $95 \%$ confidence interval 0.94 to 1.02 ). Secondary analyses revealed no differences in the risk of death or acute myocardial infarction between the two drugs, but the risk of heart failure was unexpectedly lower among patients treated with venlafaxine (hazard ratio $0.87 ; 95 \%$ CI 0.80 to 0.95 ). We found consistent results after stratification according to pre-existing cardiovascular disease.

Discussion \& Conclusion: As compared with sertraline, venlafaxine is not associated with an increased risk of adverse cardiac events in older patients.

What Is the Effectiveness of Quality Improvement Strategies on Health-Care Utilization Among Frequent Users?

J. Antony ${ }^{1}$, P.A. Khan ${ }^{1}$, N. Ivers ${ }^{2}$, E. Blondal ${ }^{1}$, M. Ghassemi ${ }^{1}$, H. Ashoor ${ }^{1}$, H. MacDonald ${ }^{1}$, L. Kark Ezer ${ }^{1}$, S.E. Straus ${ }^{1}$, A.C. Tricco ${ }^{1}$

${ }^{1}$ Li Ka Shing Knowledge Institute at St. Michael's Hospital, Toronto, ON, and ${ }^{2}$ Women's College Hospital, University of Toronto, Toronto, ON, Canada

Background/Purpose: Frequent users of emergency departments, clinics and hospitals utilize a disproportionately large amount of health-care resources, thereby reducing efficiency and decreasing overall quality of care. As such, efforts have been made to implement quality improvement (QI) strategies targeting this population. Our systematic review aims to identify effective care coordination QI strategies for frequent users.

Methods: We searched multiple databases (e.g., Cochrane Library, EMBASE, MEDLINE) from earliest date to March 2012. Additional citations were identified by scanning the reference lists of included studies. Citations and full-text articles were screened by two independent reviewers and relevant studies were abstracted and appraised for quality in duplicate using the Cochrane Effective Practice and Organization of Care tool. Random effects meta-analyses were conducted using data from randomized clinical trials (RCTs). 
Results: We screened 9564 citations and 132 full-text articles resulting in the inclusion of 44 relevant studies, including 36 RCTs. The three most commonly examined QI strategies were case management, self-management, and team changes. Nineteen studies included only patients with mental illness, while 25 included patients with other chronic illnesses. Our overall meta-analyses identified that QI strategies were effective in reducing the mean length of stay in all patients. In studies including patients with chronic illness, QI strategies effectively reduced the number of patients with emergency visits and the number hospitalized. QI strategies did not significantly reduce clinic visits or the number of patients hospitalized in studies including patients with mental illness.

Discussion \& Conclusion: QI strategies can reduce utilization in patients with chronic conditions. However, patients with mental illness may be more difficult to target with these QI strategies.

\section{What Is the Effectiveness of Wound Care Interventions?}

J. Antony, E. Cogo, A. Vafaei, P.A. Khan, A. Harrington, C. Wilson, J.D. Ivory, L. Perrier, S.E. Straus, A.C. Tricco

Li Ka Shing Knowledge Institute at St. Michael's Hospital, Toronto, ON, Canada

Background/Purpose: Acute and chronic wounds result in substantial costs to our health-care system and significantly impact quality of life. Although a number of interventions are available to treat wounds, optimal strategies for wound care remain unclear. Our objective was to identify effective wound care interventions from high-quality systematic reviews in the literature.

Methods: A search was conducted using MEDLINE, EMBASE and the Cochrane Library. Citations and full-text articles were screened in duplicate to include systematic reviews of adult patients receiving wound care. Two reviewers independently abstracted study characteristic and outcome data from the included reviews and appraised review quality using the AMSTAR tool.

Results: From the 6199 titles and abstracts and 421 fulltexts screened for inclusion, 110 systematic reviews were included. Fifty-seven reviews included meta-analyses and approximately $40 \%$ were rated as high-quality. From the highest quality meta-analyses, we identified a number of effective interventions across 5 wound types: 2-layer stockings, highcompression stockings, 4-layer bandages, elastic bandages, multi-layer high-compression, elastic high-compression, Pentoxifylline with or without compression, Cadexomer iodine, and engineered skin in patients with leg ulcers; air-fluidized beds, foam mattresses, hydrocolloid dressing, nutritional support and electrotherapy for pressure ulcers; granulocyte-colony stimulating factor, hydrogel dressing, hyaluronic acid, low-frequency/high-frequency ultrasound, and skin grafts for patients with diabetic ulcers; skin grafts and silver dressing for mixed chronic wounds; and honey for patients with burns.

Discussion \& Conclusion: Our results can be used by clinicians and patients to tailor treatment by wound type. Further analysis of this data through network; meta-analysis will be of utility to decision makers, as it will allow ranking of the effectiveness of all wound care interventions in the literature.

An Osteoporosis Knowledge Translation Tool Increased Appropriate Management in Primary Care: a MultiCentre Interrupted Time Series Analysis

M. Kastner ${ }^{1}$, A. Sawka ${ }^{1}$, M. Chen ${ }^{2}$, K. Thorpe ${ }^{3}$, C. Marquez ${ }^{4}$, D. Newton ${ }^{4}$, S. Straus ${ }^{4,5}$, M. Chignell ${ }^{6}$

Department of Medicine, Toronto General Hospital, University Health Network, Toronto, ON; ${ }^{2}$ Toronto Health Economics and Technology Assessment Collaborative, University Health Network, Toronto, ON; ${ }^{3}$ Dalla Lana School of Public Health, University of Toronto, Toronto, ON; ${ }^{4}$ Knowledge Translation Program, Li Ka Shing Knowledge Institute of St. Michael's Hospital, Toronto, ON; ${ }^{5}$ Geriatric medicine, University of Toronto, Toronto, ON; ${ }^{6}$ Department of Mechanical and Industrial Engineering, University of Toronto, Toronto, ON, Canada

Background/Purpose: Osteoporosis affects over 200 million people worldwide at a high cost to health care. Guidelines are available, but many patients are not receiving appropriate care. We developed an osteoporosis knowledge translation $(\mathrm{Op}-\mathrm{KT})$ tool to support clinical decision making: a tablet-initiated risk assessment questionnaire (RAQ), which generates best practice recommendations for physicians; and a customized education sheet for patients. We evaluated its impact on the initiation of appropriate osteoporosis disease management in primary care.

Methods: Following an implementation plan in 3 family practices in Hamilton, Ontario that included workflow analysis, the Op-KT tool was evaluated using an interrupted time series design. This involved multiple assessments 12 months before (baseline) and 12 months after introducing the tool. Analysis included segmented linear regression models and analysis of variance.

Results: Five family physicians from 3 practices participated; 2840 patients (mean age 67 years; $79 \%$ women) were eligible (31\% of the practice population). Time series regression models showed an increase from baseline in the 
initiation of bone mineral density testing $(3.2 \% ; p=.02)$, any osteoporosis medication $(0.5 \% ; p=.0064)$, and calcium or vitamin $\mathrm{D}(1 \%, p=.0013)$. The RAQ was completed without prompting by 351 patients (mean age 64 years; $77 \%$ women; mean time to completion 3.43 minutes). Of these, 276 patients $(79 \%)$ were at risk for osteoporosis (1 major or 2 minor risk factors).

Discussion \& Conclusion: Our multi-component Op-KT tool significantly increased osteoporosis investigations in 3 family practices. The study highlights the potential of using decision support tools at the point of care in busy, short-visit practices to facilitate patient self-management.

\section{Improving Care for Hip Fracture Patients-a Canadian Initiative (Bone and Joint Canada)}

\section{A. Byszewski ${ }^{1}$, R. McGlasson ${ }^{2}$, J. Waddell ${ }^{3}$ \\ ${ }^{1}$ The OttawaHospital, Civic Campus, Ottawa, ON; ${ }^{2}$ Bone and Joint Canada, Toronto, ON; ${ }^{3}$ Orthopedic Surgery, University of Toronto, Toronto, ON, Canada}

Background/Purpose: Almost 30,000 patients annually experience a hip fracture in Canada. They tend to be older, frail with multiple chronic illnesses, including a high incidence of dementia and delirium. For many, the hip fracture results in poor outcomes including loss of function and use of ALC (alternate level of care) beds. In 2011 Bone and Joint Canada (BJC) worked with health-care professionals from across the country to develop a National Hip Fracture Toolkit, which was based on available evidence and a consensus building approach, to provide clinical and system best practices to better manage these patients and return them home.

Methods: A knowledge translation approach was used to assist provinces to review their performance and to facilitate uptake of best practices. Identified barriers to care included the management of frail patients and their co-morbidities, access to rehabilitation, weight bearing, and patient education.

Results: All provinces participated in the KT strategy at a national level, as well as hosting provincial and local events to measure their performance against the Toolkits recommendations. Care maps were implemented at a provincial level, and local improvement initiatives were undertaken in all provinces in 2012/2013. Surgeon practices to promote weight bearing were investigated and patient education materials were developed. In Ontario, recommendations on best practice were made for future funding of hip fracture patients.

Discussion \& Conclusion: The National Toolkit provides a system and clinical practice information on pre-operative, surgical, and post-operative care. It uses a multidisciplinary and multi-faceted approach to the clinical and operational management of older hip fracture patients and has improved care across the country.

\section{In-Patient Dementia Screening at Southend University Hospital}

S. Faber ${ }^{1}$, I. Liakas ${ }^{1}$, B. Maddock ${ }^{1}$, C. Timms 2 , J. Ling ${ }^{2}$

${ }^{I}$ Medicine for the Elderly, Southend-On-Sea, Essex, and

${ }^{2}$ Memory Service, Southend-On-Sea, Essex, UK

Background/Purpose: The UK Commissioning for Quality and Innovation Dementia (CQUIN) framework (2012) aims to facilitate early identification of patients with dementia during their inpatient stay and ensure they are referred to appropriate services. Step 1 is to find all patients over age 75 years meeting the inclusion criteria. Step 2 is assessment using a screening questionnaire, AMTS, collateral history, examination, and investigations, in order to stratify all patients as suspected dementia, known dementia or no cognitive impairment. Step 3 is referral to memory services or GP for further follow-up. We aimed for rapid assessment of all patients over 75 years of age admitted acutely to achieve 90\% compliance in Steps 1-3.

Methods: All patients over 75 were identified on a daily basis. A pro forma was developed and available in all wards. Junior physicians in elderly care wards assessed patients during daily rounds. The roles of the memory nurses were changed to supervise data collection and review patients in other wards.

Results: The total number of patients assessed in September was 341/377 (90.5\%) and October 2012 465/494 (97\%). Of these patients with a diagnosis of delirium or who scored positively on the screening question, 113/113 (100\%) and 192/198 (97\%), respectively, had a dementia diagnostic assessment. In September 54/55 (98.2\%) and October 133/133 (100\%) of patients with suspected dementia were referred for further follow-up who might have been missed with standard care.

Discussion \& Conclusion: Implementation of the UK Dementia (CQUIN) framework is achievable through staff education, change in working practice, and clear implementation of protocols, with little extra resources. Early recognition of suspected dementia increases early access to appropriate support for patients and their careers.

Palliative Care and its Impact on the Aggressiveness of End-of-Life Care in Older Patients with Advanced Pancreatic Cancer 
R. Jang, M. Krzyzanowska ${ }^{1}$, C. Zimmermann ${ }^{1}$, N. Taback ${ }^{2}$, S. Alibhai ${ }^{3}$

${ }^{1}$ Princess Margaret Hospital, Toronto, ON; ${ }^{2}$ University of Toronto, Toronto, ON; ${ }^{3}$ University Health Network, Toronto, ON, Canada

Background/Purpose: Our objective was to examine the impact of specialized palliative care (PC) (defined as a physician consultation focusing on $\mathrm{PC}$ needs, lasting at least 40 minutes) for adults 70 and older on: (a) use of chemotherapy within 14 days of death, (b) more than one emergency department (ED) visit, (c) more than one hospitalization, and (d) at least one intensive care unit (ICU) admission, all within 30 days of death.

Methods: A retrospective population-based cohort study using linked administrative databases in Ontario was conducted with patients diagnosed with advanced pancreatic cancer from 1 Jan 2005 to 31 Dec 2010. Multivariable logistic regression analyses were performed with the above quality indicators as the outcomes of interest and PC as the exposure, adjusting for other variables (age, sex, comorbidity, rurality, and health region).

Results: Of 6,076 patients with advanced pancreatic cancer, $58 \%$ were age 70 or older, and 5,381 had died at last follow-up. $57 \%(1251 / 2187)$ of those younger than 70 and $49 \%(1565 / 3194)$ of those 70 and older received a PC consultation $(p=.0001)$. PC was associated with a lower odds (OR) of aggressive care among all age groups: $0.34(95 \% \mathrm{CI} 0.25-0.46)$ for chemotherapy; 0.12 (95\% CI 0.08-0.18) for ICU; 0.19 (95\% CI 0.16-0.23) for multiple ED visits; and 0.24 (95\% CI 0.19-0.31) for multiple hospitalizations near death. Older age was also associated with lower odds of aggressive care for all four outcomes.

Discussion \& Conclusion: In patients with advanced pancreatic cancer, $\mathrm{PC}$ is associated with less frequent aggressive care across all age groups, but $\mathrm{PC}$ consultation and aggressive care were both less likely in older patients (70+).

Evaluating the Impact of IMPACT: Protocol for an RCT of an Interprofessional Team Intervention for Older Patients with Multimorbidity

J. Nie ${ }^{1}$, L. Wang ${ }^{1}$, L. Nickell ${ }^{2}$, J. Charles ${ }^{2}$, H. Abrams ${ }^{3}$, R. Upshur $^{1}$, S. Tracy ${ }^{1}$

${ }^{I}$ Primary Care Research Unit, Sunnybrook Health Sciences Centre, Toronto, ON; ${ }^{2}$ Department of Family and Community Medicine, Sunnybrook Health Sciences Centre, Toronto, ON; ${ }^{3}$ University Health Network, Toronto General Hospital, Toronto, ON, Canada

Background/Purpose: To meet the challenges of population aging and increasing multimorbidity, significant reform to health-care systems is underway. New models of care include the patient-centred medical home and interprofessional teambased approaches; however, there has been limited exploration of the effectiveness of such interventions for patients with multimorbidity. To evaluate both the clinical-effectiveness and cost-effectiveness of a team-based model of primary care specifically designed for older patients with multimorbidity.

Methods: Multi-site randomized controlled trial of the IMPACT clinic (Interprofessional Model of Practice for Aging and Complex Treatments). Inclusion criteria: patients aged $65+$, three or more chronic diseases requiring monitoring and treatment, five or more long-term medications, and minimum of one functional ADL limitation. Exclusion criteria: home-bound or institutionalized patients, or deemed unsuitable by the usual family physician. The IMPACT team comprises family physicians, specialist physicians, visiting nurse, pharmacist, community social worker, occupational therapist, physiotherapist, dietitian, and care navigator. IMPACT patients are scheduled for extended visits (1.5 to 2 hours) during which the full team works collaboratively with the patient and family caregiver(s) to address current medical, functional, and psycho-social issues. During the visit, a pro-active interprofessional care plan is developed, a comprehensive medication review is conducted, and a discussion of anticipatory care planning is initiated.

Results: Primary outcomes include Emergency Department visits, hospitalizations, and physician visits. Secondary outcomes include patient satisfaction, family caregiver satisfaction, provider satisfaction, quality of life, caregiver strain, and interprofessional team function.

Discussion \& Conclusion: This RCT will contribute muchneeded evidence on the effectiveness of a team-based primary care intervention for older patients with multimorbidity.

An Update on a Systematic Review of the Use of Geriatric Assessment for Older Adults in Oncology

M. Puts ${ }^{1}$, B. Santos ${ }^{1}$, J. Hardt ${ }^{1}$, J. Monette ${ }^{2}$, V. Girre ${ }^{3}$, E. Springall ${ }^{4}$, S. Alibhai ${ }^{5}$

${ }^{1}$ Lawrence S. Bloomberg Faculty of Nursing, University of Toronto, Toronto, ON, Canada; ${ }^{2}$ Division of Geriatric Medicine, Jewish General Hospital, Montreal, QC, Canada; ${ }^{3}$ Department of Medical Oncology-Hematology, Centre Hospitalier Departemental, La Roche sur Yon, NA, France; ${ }^{4}$ Gerstein Information Science Centre, University of Toronto, Toronto, ON, Canada, ${ }^{5}$ Department of Medicine, University Health Network, Toronto, ON, Canada

Background/Purpose: Cancer is a disease that mostly affects older adults. A geriatric assessment (GA) has been 
recommended for older adults to assist with treatment decision-making. The aims of review: 1) to provide an overview of the use of GA in oncology; 2) to examine feasibility and psychometric properties; 3) to systematically evaluate the effectiveness of GA in predicting/modifying outcomes.

Methods: A systematic review of literature published between November 2010 and July 2012. Articles published in 5 databases in English, Dutch, French, and German were included. Articles were selected and reviewed by 2 independent reviewers.

Results: 34 manuscripts reporting on 33 studies were identified. The quality of most studies was moderate to good. Of all studies, 14 were prospective, 8 cross-sectional, 5 retrospective and 7 others (mostly phase II trials of a new treatment regimen). A GA was shown to be feasible, the time needed to complete ranged between 5 and 120 minutes, were mostly conducted in the outpatient oncology setting by nurses, and most often included the domains activities of daily living, co-morbities, cognitive function, depression, medications, and fall risk assessment. Four studies examined psychometric properties of the GA with satisfactory results, and 18 studies examined the predictive ability of the GA and showed that components of the GA predicted treatment toxicity and overall survival.

Discussion \& Conclusion: Although the studies showed that GA was feasible and had predictive validity, there has not been a randomized controlled trial showing the effectiveness of the GA in improving outcomes for older adults with cancer.

\section{Turning Back the Clock: Rejuvenating Fracture Healing}

L. Vi, G. Baht, B.A. Alman

Developmental and Stem Cell Biology, Hospital for Sick Children, Toronto, ON, Canada

Background/Purpose: The capacity for bone repair and regeneration diminishes with age. This prolongs fracture healing time and, in some instances, results in non-union, requiring extensive surgery. The mechanism behind this is not known; however, studies thus far have assumed the reason to be a decrease in the capacity of bone marrow mesenchymal stem cells (MSCs) to differentiate into bone-forming cells (osteoblasts). We found that old MSCs can be "rescued" to behave like young MSCs when cultured in media pre-treated by young cells. These findings implicate the presence of a "youth factor" that is secreted by young bone marrow cells and is able to rescue the aged phenotype of old cells. Thus, the purpose of this study is to determine the cell type responsible for rescue of osteoblast differentiation in old cells, and to determine its effects on fracture repair in old mice.
Methods: Bone marrow cells were isolated from young and old mice. Osteoblast differentiation in culture was determined by quantifying colony forming unit-osteoblast. Fracture repair was assessed using a tibial fracture mouse model.

Results: Co-culture of old cells with young hematopoietic stem cells (HSCs) promoted osteoblast differentiation of these old cells. Interestingly, an adherent F4/80+ cell population (a marker of monocyte-macrophage cell lineage) was identified in young, but not old, HSC cultures. In culture, exposing old MSCs to media pre-treated by young macrophages induced osteoblast differentiation of these cells. Furthermore, bone marrow transplantation of young F4/80+ cells into old mice resulted in improved fracture repair.

Discussion \& Conclusion: This study demonstrates that young macrophages secrete soluble factors that can rescue osteoblast differentiation and improve fracture repair in older animals.

\section{What Do We Know About the Patients Who Are Alternate Level of Care in Horizon Health Network?}

P. Jarrett ${ }^{1}$, R. McCloskey², A. McCollum ${ }^{3}$, H. Oakley ${ }^{4}$, C. Stewart ${ }^{5}$

${ }^{I}$ Department of Geriatric Medicine, Horizon Health Network, Saint John, NB; ${ }^{2}$ Nursing and Health Sciences, University of NB Saint John, Saint John, NB; ${ }^{3}$ Dalhousie University, Saint John, NB; ${ }^{4}$ Health and Aging Program, Horizon Health Network, Saint John, NB; ${ }^{5}$ Department of Computer Science and Applied Statistics, University of NB Saint John, Saint John, NB, Canada

Background/Purpose: The Canadian Institute of Health Information (CIHI) designates patients who remain in hospital after their acute care phase is completed as Alternate Level of Care (ALC) patients. Understanding who the ALC population is in hospital is needed.

Methods: All ALC patients Horizon Health Network (HHN) as of Feb 9, 2012 were identified. A data collection tool, designed for the study, was used to review charts.

Results: There were 413 ALC patients identified, occupying $25.2 \%$ of all hospital beds within HHN. A stratified random sample from 7 hospitals comprised the sample of 223. Two were excluded due to long length of stays, giving a sample of 221.The mean age was 78.4 years. Prior to admission, 51 (23.1\%) were living in a care institution in the community. Dementia was a diagnosis in $53.9 \%$. The overall mean length of stay until data collection was 293.4 days. Six months later, $81(36.7 \%)$ were still in hospital, $32(14.5 \%)$ had died, and $65(29.4 \%)$ were discharged to nursing home. For those 
discharged to a nursing home, the mean length of stay was 262.8 days. For those still in hospital, the mean length of stay was 683.9 days.

Discussion \& Conclusion: The majority of ALC patients are elderly with dementia. Six months after data collection, the majority remain in hospital with a mean length of stay of almost 2 years. Even those who went to a nursing home, the length of stay was almost 9 months.

Do Quality of Life and Physical Function at Diagnosis Predict Short-Term Outcomes During Intensive Chemotherapy in Younger or Older Acute Myeloid Leukemia Patients?

S. Alibhai ${ }^{5}$, N. Timilshina ${ }^{1}, \mathrm{H}$. Breunis $^{1}$, M. Minden ${ }^{2}$, V. Gupta $^{2}$, M. Li ${ }^{3}$, G. Tomlinson ${ }^{1}$, R. Buckstein ${ }^{4}$, J. Brandwein ${ }^{2}$ ${ }^{1}$ Department of Medicine, University Health Network (UHN), Toronto, ON; ${ }^{2}$ Department of Medical Oncology and Hematology, UHN, Toronto, ON; ${ }^{3}$ Department of Psychiatry, UHN, Toronto, ON; ${ }^{4}$ Department of Medical Oncology and Hematology, Sunnybrook Health Sciences Centre, Toronto, ON; ${ }^{5}$ Department of Medicine, Division of Internal Medicine and Geriatrics, UHN, Toronto, ON, Canada

Background/Purpose: Identifying measures to predict short-term toxicities in patients undergoing intensive chemotherapy (IC) for acute myeloid leukemia (AML) is needed. Emerging data suggest that quality of life (QOL) assessment and/or physical performance measures (PPMs) may predict outcomes in oncology, although there are no data in AML patients.

Methods: We conducted a prospective, longitudinal study of adults (age 18-59) and older (age 60+) AML patients undergoing IC. Prior to starting IC, patients completed the EORTC QLQ-C30 and FACT-Fatigue, in addition to PPMs (grip strength, timed chair stands, and 2-minute walk test). Outcomes included 60-day mortality, intensive care unit (ICU) admission, and achievement of complete remission (CR). Logistic regression was used to evaluate each outcome.

Results: Of the 243 patients (median age $57.5 \mathrm{yrs),56.7 \%}$ were male, and 96 (40\%) were older. 60-day mortality, ICU admission, and CR occurred in 9 (3.4\%), 15 (6.2\%), and 171 (70.4\%), respectively. In univariate regressions, neither QOL nor PPMs were predictive of 60-day mortality (all $p>.05$ ), whereas cytogenetic risk group $(p=.04)$, ICU admission $(p \leq .001)$, and remission status at 30 days $(p=.006)$ were. Fatigue was a significant predictor of ICU admission $(p=$ .02 ), whereas QOL and baseline PPMs were not. In univariate analyses, higher Charlson score was a significant predictor of both ICU admission $(p=.01)$ and remission status at 30 days $(p=.002)$. Neither QOL nor PPMs were predictive of achieving CR (all $p>.05$ ). Findings were similar among the subset of older patients.

Discussion \& Conclusion: Baseline QOL and PPMs were not associated with short-term mortality, ICU admission, or achievement of CR after the 1st cycle of chemotherapy for AML.

The Relationship of Self-Rated Health with Measures of Functional Status and Mortality: Results of a Prospective Pilot Study with Older Newly-Diagnosed Cancer Patients

M. Puts ${ }^{1}$, J. Monette 2 , V. Girre ${ }^{3}$, C. Wolfson ${ }^{4}$, M. Monette ${ }^{5}$, G. Batist ${ }^{6}, \mathrm{H}$. Bergman

${ }^{1}$ Lawrence S. Bloomberg Faculty of Nursing, University of Toronto, Toronto, ON, Canada; ${ }^{2}$ Division of Geriatric Medicine, Jewish General Hospital, Montreal, QC, Canada; ${ }^{3}$ Department of Oncology-Hematology, Centre Hospitalier Departemental, La Roche Sur Yon, NA, France; ${ }^{4}$ The Research Institute of McGill University Health Centre, Montreal, QC, Canada; ${ }^{5}$ Solidage Research Group on Frailty and Aging, Jewish General Hospital, Montreal, QC, Canada; ${ }^{6}$ Segal Cancer Centre, Jewish General Hospital, Montreal, QC, Canada; ${ }^{7}$ Department of Family Medicine, McGill University, Montreal, QC, Canada

Background/Purpose: Self-rated health (SRH) has been shown to predict functional status in older adults, but this has less often been examined for older cancer patients. The aim of this study was to determine the association between SRH and functional status, comorbidity, toxicity of treatment, and mortality in older newly diagnosed cancer patients.

Methods: Patients aged 65 and over, newly diagnosed with cancer, recruited at the Jewish General Hospital. SRH was evaluated prior to treatment, and at 3, 6, and 12 months. Functional status (Instrumental Activities of Daily Living (IADL), Basic Activities of Daily Living (ADL), ECOG Performance Status (ECOG PS), and frailty markers (low grip strength, mobility impairment, physical inactivity, cognitive impairment, mood impairment, and poor nutritional status) were measured at baseline, 3 , and 6 months. Treatment toxicity and mortality were abstracted from the chart. Chi-square tests and $t$-tests were used to compare patients who rated their SHR as fair/poor/very poor to those very good/good with regard to functional status, frailty, and co-morbidity. Logistic and Cox regression were used to examine the association between baseline SRH and treatment toxicity/time to death.

Results: There were 112 participants, median age 74.1. At baseline, $74(66.1 \%)$ had a good SRH and $38(33.9 \%)$ had poor SRH and those had more co-morbidities, more frailty 
markers present, lower ECOG PS and IADL impairments. We found no association between SRH and toxicity or mortality.

Discussion \& Conclusion: There was a moderate correlation between SRH and the number of frailty markers, IADL disability, and co-morbidities, but SRH did not predict toxicity or mortality.

\section{A Novel Community Paramedicine Initiative in Toronto}

Amol Verma ${ }^{1}$, Adam Thurston ${ }^{1}$, Cindy Nicholson ${ }^{1}$, Paul Raftis $^{1}$, Samir Sinha ${ }^{2}$

${ }^{1}$ Toronto EMS, Toronto, ON, and ${ }^{2}$ Geriatrics, Mount Sinai Hospital, Toronto, ON, Canada

Background/Purpose: People over the age of 60 account for $60 \%$ of paramedic responses in Canada. Many of these calls are not life-threatening or time-sensitive. Paramedics have a unique opportunity to engage people in their homes and they often see people in vulnerable circumstances. The field of Community Paramedicine is growing in Ontario and across Canada due to its potential to provide alternative patient care pathways. In 2006, Toronto Emergency Medical Services (EMS) established its Community Referrals by EMS (CREMS) program to link patients with Community Care Access Centres (CCAC). With a simple phone, call paramedics identify and connect patients with community support services.

Methods: To determine the effectiveness of the Community Paramedicine program, 904 patients referred to CCACs in 2011 were evaluated for improved outcomes and reduced reliance on EMS. The 6-month period prior to initiating the CCAC referral was compared to the 6 months post-CCAC referral.

Results: The total calls to EMS were reduced from 2,715 to 1,340 for this patient group. Transports to emergency departments also decreased from 1654 to 582 . Paramedics spent less time overall with these patients, reduced from 4597.28 hours to 1898.87 hours. Based on the decreased transports and time spent assessing and treating these patients, cost savings have been estimated to be as high as $\$ 321,600.00$ for the 6-month post-referral period.

Discussion \& Conclusion: Community Paramedicine offers an innovative, cost effective opportunity to improve the health care of elders wishing to age and live at home independently. Future directions will include exploring an expanded scope of clinical, practice for paramedics, and a more systematic evaluation of the CREMS program with an eye toward broader implementation.
Quality of Care Provided to Prostate Cancer (PCa) Elderly Patients for Prevention and Treatment of Osteoporosis Induced by Androgen Deprivation Therapy (ADT): Results of a Chart Audit

Rehab Chahin, Shabbir Alibhai, Henriette Breunis

Department of Medicine, University Health Network, Toronto, ON, Canada

Background/Purpose: Men with PCa on ADT are at risk of decreased bone mineral density (BMD) and osteoporosis. Guidelines recommend referral to specialized clinics, but the quality of care in osteoporosis clinics and benefits to patient have never been reported.

Methods: Charts for 67 men (mean age 74.2 yrs) on ADT referred to an academic osteoporosis clinic between 2010 and 2011 were reviewed. The following quality of care issues were examined: (a) services provided to $\mathrm{PCa}$ patients receiving ADT (e.g., screening, preventing, and treating osteoporosis); (b) use of Canadian guidelines to target appropriate therapies.

Results: $56(83.6 \%)$ received continuous ADT for a mean of $27.4 \pm 30.7$ months at the baseline visit. $37(55.2 \%)$ had osteopenia and 15 (22.4\%) had osteoporosis. At initial consultation, $55.2 \%$ were taking $1000 \mathrm{mg}$ calcium daily from all sources, while $26.9 \%$ were taking more than $1200 \mathrm{mg} ; 22.4 \%$ were taking Vitamin D 3 months. For lifestyle recommendations, $71.4 \%$ of sedentary patients were advised to increase exercise. Of the 39 (70\%) and 24 (39.2\%) patients who were not taking appropriate amounts of calcium and vitamin D, respectively, $100 \%$ were recommended to adjust their intake to guideline levels.

Discussion \& Conclusion: The osteoporosis clinic performed a comprehensive assessment and recommended guideline-based bone health care for the vast majority of men on ADT, suggesting a systematic approach to assessing bone health is associated with high rates of guideline-adherent care.

\section{Does Frailty Impact Geriatric Outcomes in Older Men with Prostate Cancer Undergoing Chemotherapy?}

Salman Aziz ${ }^{1}$, Tharsika Manokumar ${ }^{1}$, Faraz Rizvi ${ }^{1}$, Henriette Breunis $^{1}$, Anthony Joshua ${ }^{2}$, Ian Tannock ${ }^{1,2,3}$, Shabbir M.H. Alibhai $^{1}$

${ }^{1}$ University Health Network, Toronto, ON; ${ }^{2}$ Division of Medical Oncology and Hematology, Princess Margaret Cancer Centre, Toronto, ON; ${ }^{3}$ Department of Medicine, University of Toronto, Toronto, ON, Canada

Background/Purpose: Metastatic castration-resistant prostate cancer (mCRPC) is characterized as disease progression 
despite adequate androgen deprivation therapy (ADT). Although chemotherapy for mCRPC prolongs survival, whether its impact on elderly-relevant outcomes and toxicity differ by frailty status is not known.

Methods: Men aged 65+ with $\mathrm{mCRPC}$ who were starting first-line chemotherapy were enrolled in this longitudinal prospective pilot study. Elderly-relevant information was collected at baseline and before the start of each chemotherapy cycle. Frailty was assessed by the Vulnerable Elders Survey (VES-13), functional status by OARS-IADL, social activities limitation and support by MOS measures, and FACT-G and FACT-P for general and prostate-specific quality of life (QOL), respectively. Physical function was assessed by timed up and go (TUG), timed chair stands, and grip strength. Changes in outcomes were analyzed between frail vs. nonfrail patients using Student's $t$-test and linear regression.

Results: 21 patients (mean age 74), of whom 11 were frail (VES-13 3), were assessed. Generally, at baseline frail patients were slightly older and scored lower than non-frail patients in QOL, functional status, physical function, and social support and activities. However, frail patients improved more than non-frail patients in all domains, except TUG. 18\% of frail patients died during the course of therapy compared to no deaths in non-frail patients.

Discussion \& Conclusion: Frail patients, as determined by VES-13 3, with mCRPC may represent a heterogeneous population; one group destined to die soon and the other who may do well with chemotherapy. Further research and patient recruitment is needed to determine whether a subset of frail older patients would benefit from first-line chemotherapy treatment.

Examining the Accuracy of the Clock Drawing Task for Assessing Driving Competency of Cognitively Impaired Drivers

Jean Triscott, Elizabeth Triscott, Bonnie Dobbs

Department of Family Medicine, University of Alberta, Edmonton, AB, Canada

Background/Purpose: The Clock Drawing Test (CDT) is a screening tool used by physicians for detecting dementia in the clinical setting and is commonly used for identifying drivers with a dementia whose driving skills may have declined to an unsafe level. However, the accuracy of the CDT for detecting declines in driving due to a dementia is not well-established and is confounded by the presence of multiple scoring systems. The purpose of the study was to examine the intra-rater reliability of a novice scorer; the inter-rater reliability between a novice scorer and a trained clinician; and the relationship between different CDT scoring methods and on-road driving performance.

Methods: 50 cognitively impaired and cognitively intact participants completed the CDT and an on-road assessment. A novice scorer and a trained clinical geriatric specialist scored the clocks using 4 CDT scoring systems (Rouleau, Shulman, Freund, and MoCA).

Results: The intra-rater reliability of the novice scorer across the four scoring schemes was high (Pearson's $r$ of 0.85 to 0.90 , all $p=.01$ ), as was the inter-rater reliability between the Novice Scorer and the Geriatric Specialist (Pearson's $r$ of 0.68 to 090 , all $p=.01$ ). None of the CDT scores were significantly related to on-road outcomes.

Discussion \& Conclusion: Although there was good intraand inter-rater reliability for the scoring systems tested, none of the CDTs examined were significantly associated with on-road outcomes, indicating that use of CDT scores is most likely to result in erroneous driving decisions for cognitively impaired patients.

Functional Gains and Discharge Destinations of Patients Admitted to a Geriatric Slow Stream Rehabilitation Unit

Paul Katz ${ }^{1}$, Anna Berall ${ }^{2}$, Gary Naglie ${ }^{1}$, Angela Chan ${ }^{3}$, Jurgis Karuza $^{2}$, Grace Leung ${ }^{4}$

${ }^{I}$ Department of Medicine, Baycrest, Toronto, ON; ${ }^{2}$ Program Evaluation Unit, Baycrest, Toronto, ON; ${ }^{3}$ Department of Rehabilitation, Baycrest, Toronto, ON; ${ }^{4}$ University of Toronto, Toronto, ON, Canada

Background/Purpose: In 2010, Baycrest implemented a Slow Stream Rehabilitation Program (SSR) to deliver a lowintensity long-duration rehab for frail seniors' post-acute hospitalization. To examine the change in function, length of stay, and discharge destination of patients admitted to SSR.

Methods: Psychosocial and functional measures were administered to patients on admission and discharge to the SSR Unit.

Results: Over a period of 15 months, 105 patients ( $70 \%$ of all admissions) were recruited; mean age was 82 , mean stay in acute care was 32 days, and the mean LOS in SSR was 88 days. On admission, $85 \%$ had mild/moderate to severe cognitive impairment (MoCA: 26 ) and $78.5 \%$ were dependent with transfers with or without devices. Mean admission FIM: 51 and discharge FIM: 74; admission Berg Balance Scale (BBS): 10 and discharge BBS: 19.7. On admission 51\% could ambulate 10 steps with a device and $80.4 \%$ on discharge. Upon discharge, $68 \%$ were discharged home or to other 
community residences; $24 \%$ to Long-Term Care (LTC) and $9 \%$ went to acute care.

Discussion \& Conclusion: This study confirms that the SSR population is a frail elderly group admitted after a mean of 32 days in acute care. With low functional ability on admission, this group was able to achieve over $80 \%$ ambulation with or without a device and had a mean discharge FIM of 74. After 88 days of low-intensity rehab, $68 \%$ were able to return to community living. After a long acute hospital stay, frail older adults with cognitive impairment can benefit from slow stream rehabilitation to prepare them for living in the community rather than going to LTC.

\section{Preceptors' Perspectives on International Medical Gradu- ate (IMG) Residents Becoming Culturally Competent Within the Canadian Medical Context}

Jean Triscott ${ }^{1}$, Olga Szafran ${ }^{1}$, Earle Waugh ${ }^{2}$

${ }^{I}$ Division of Care of the Elderly, Department of Family Medicine, University of Alberta, Edmonton, AB, and ${ }^{2}$ Centre for the Cross-Cultural Study of Health and Healing, University of Alberta, Edmonton, AB, Canada

Background/Purpose: Residents who are international medical graduates (IMGs) are a heterogeneous group of learners with distinct backgrounds of ethnicity, religion, and culture. They came from various countries with differing medical education standards, societal values, and professional codes of conduct. When training and working in Canada, IMG residents may experience trans-cultural challenges. The purpose of this study is to identify cultural strengths and challenges that IMG family medicine residents encounter when working and training within the Canadian medical context, and to identify the values, behaviours, and codes of conduct expected of family physicians working in Canada.

Methods: Focus group with seven academic/community preceptors who teach residents. Qualitative data were transcribed and analyzed for emerging themes.

Results: Distinctive Canadian socio-medico-cultural values were identified in six theme areas - communication, gender, cultural awareness, ethics, medical knowledge, and social hierarchy. IMG residents were noted to possess strengths in: ability to speak multiple languages; establishing rapport with patients of a similar culture; understanding culturally-defined gender roles; knowledge of global diseases; skilled at procedures; proficient in making diagnoses based on clinical indicators; and possessing a sense of responsibility to the greater community. The challenges that IMG residents were noted to encounter include: difficulty with language nuances; culturally-defined gender interactions; challenges of dealing with patients from diverse cultural groups; limited understanding of ethics; disease-focused care; hierarchical/didactic approach to learning; and tendency not to ask questions during the learning process.

Discussion \& Conclusion: Cultural gaps appear to be present when IMG residents interface within the Canadian medical context. Identification of trans-cultural challenges will assist in the development of teaching resources for use in IMG resident training.

Development and Implementation of an Interdisciplinary Pain Management Model for Older Adults Living in Long-Term Care

Haley Weber ${ }^{1}$, Ramesh Zacharias ${ }^{1}$, Carlos Rojas-Fernandez ${ }^{2}$ ${ }^{1}$ Schlegel-UW Research Institute for Aging, Kitchener, ON, and ${ }^{2}$ University of Waterloo School of Pharmacy, Kitchener, ON, Canada

Background/Purpose: The purpose of this study is to develop a novel interdisciplinary pain management (IPM) model to better treat and manage pain within the elderly population residing in long-term care institutions.

Methods: This project is being carried out as a multiphase study:

1. Medical record review of 180 patient charts characterizing the usual care model currently relied upon in representative facilities.

2. One-on-one staff oriented interviews discussing staff perceived barriers, challenges and strengths concerning current pain management practices. Grounded theory will be utilized to analyze transcripts and develop theories.

3. Focus group session aimed at further exploring themes developed during one-on-one interviews.

4. Details of the interdisciplinary model will be delineated. This phase will encompass creation of all educational materials, tools, and standard operating procedures.

5. Implementation of model will take place via comparison study. A cohort of residents will have pain scores measured before (usual care) and following implementation of interdisciplinary pain management model.

Results: An interdisciplinary pain management model for patients in long-term care facilities is established. Implementation and trialing of the interdisciplinary model will prove to be more beneficial than the standard care model. Ultimately, this will be demonstrated by an overall improvement in resident pain scores.

Discussion \& Conclusion: The development and utilization of an interdisciplinary pain management model will 
provide a useful and efficacious method to treat pain in the aged living within long-term care facilities.

\section{The Impact Clinic: an Innovative Model of Interprofes- sional Care for Elderly Patients with Complex Health- Care Needs}

Shawn Tracy ${ }^{1}$, Stephanie Bell ${ }^{1}$, Leslie Nickell ${ }^{2}$, Jocelyn Charles $^{2}$, Ross Upshur ${ }^{2}$

${ }^{1}$ Primary Care Research Unit, Sunnybrook Health Sciences Centre, Toronto, ON, and ${ }^{2}$ Department of Family and Community Medicine, Sunnybrook Health Sciences Centre, Toronto, ON, Canada

Background/Purpose: The growing number of elderly patients with multiple chronic conditions presents a pressing challenge to the Canadian health-care system. Current practice models are not well suited to this challenge. Our primary objective was to design and evaluate a new interprofessional care model for community-dwelling seniors with complex health-care needs. A secondary objective was to explore the potential of the new model as an interprofessional training opportunity.

Methods: The IMPACT clinic (Interprofessional Model of Practice for Aging and Complex Treatments) features an extended visit (90 minutes) with a comprehensive interprofessional team. The model is designed to be patient-centred and family-friendly and attempts to bridge primary care, specialty care, and community care. IMPACT was pilot-tested at one site and peer-modeled at three other sites. A multi-method evaluation included a chart audit, survey of team function, and qualitative interviews with patients/families.

Results: Observed benefits of the IMPACT clinic include: significantly more time and "space" for the patient and family to discuss current concerns; reduction in repeat visits and multiple referrals; enhanced real-time information-sharing; improved professional understanding of other disciplines; greater satisfaction among health-care providers; and enhanced interprofessional learning among clinical trainees. Challenges included: extended length of visits proved exhausting for some frail patients; interprofessional team-based models perhaps not optimal for patients with sensory impairments or severe mental health concerns; and scheduling issues sometimes arose owing to the number of clinicians involved.

Discussion \& Conclusion: Evaluation of the IMPACT clinic is encouraging with positive feedback from patients/ families, team members, and clinical trainees. Interprofessional care models hold great promise for meeting the challenge of complex chronic disease in the elderly. Further evaluation is underway.

\section{Learning Needs Survey of Medical Directors in ON LTC Homes}

\section{Andrea Moser \\ Department of Family and Community Medicine, University of Toronto, Toronto, ON, Canada}

Background/Purpose: Medical Directors in LTC homes in Ontario are increasingly being faced with adminstrative needs of a more complex patient population and in an environment of increased legislative and regulatory oversight. There are roles identified within the LTC Homes Act, as well as key roles outlined in Medical Director Contracts agreed to by MOHLTC and the OMA. The Ontario Long Term Care Physicians is a non-profit organization with close to 300 members who are physicians working in LTC homes in Ontario. The organization runs a clinically focused conference each fall and increasingly is aware of administrative skills and expertise for which many members may not have received formal training. In addition, we hear from members challenges they face with being informed of important system changes and new programs being implemented. The purpose of the survey was to identify perceived and unperceived learning needs of physicians working in Long Term Care to explore future educational initiatives.

Methods: Unrestricted grant received from Pfizer to develop a LTC physician survey and begin developing educational initiatives based on outcomes of the survey. Survey questionnaire developed with input from OLTCP board member working group. Survey was circulated via OLTCP database. Survey results then analysed and presented to OLTCP board and membership.

Results: Survey identified perceived and unperceived learning needs in areas of legislative requirements, quality improvement, program management, high-risk clinical areas, and working with teams. Barriers to involvement in areas of administration included time and knowledge, not lack of interest. The details of these results will be shared in the poster format.

Discussion \& Conclusion: Survey identified key learning needs that are facing medical directors in LTC homes that are integral to the role of Medical Director. The OLTCP has explored training programs and conferences in North America and has determined that the content areas of the Core Curriculum on Medical Direction in LTC run by the American Medical Directors Association in the United States best matches the learning needs we have identified. We have now developed goals and objectives for an equivalency curriculum, and are in the process of developing the curriculum to address medical direction and leadership skills required to be an effective medical director in LTC. 
The "Provincialization" of the Covenant Health DecisionMaking Capacity (DMC) Model

Jasneeet Parmar' ${ }^{1}$, Suzette Bremault-Phillips ${ }^{2}$

${ }^{I}$ Alberta Health Services, Edmonton, AB, and ${ }^{2}$ University of Alberta, Edmonton, AB, Canada

Background/Purpose: As the life expectancy and chronicity of health conditions affecting Canadians continues to rise, the assessment of autonomous decision-making capacity becomes an issue of increasing importance. Adults with diseases and disabilities are at particular risk in this regard. Comprehensive assessments and realistic interventions that employ the least intrusive and least restrictive measures possible have been determined to be the most ethical and desirable.

Methods: The inter-disciplinary DMC Model was developed based on a literature search, environmental scan, needs assessment, surveys, and discussions with inter-disciplinary groups at various health-care sites within Covenant Health in 2006. An iterative process was used to formulate a model, which was then implemented in the Covenant Health and AHS sites, Edmonton zone, from 2007-2012.

Results: This model was "provincialized" through the AHS Seniors Health Cognitive Strategic Planning Committee and has been made available for use provincially. It includes a care map, worksheets, and staff training workshops and inservices, and an inventory of educational materials. Staff trained in the assessment of decision-making capacity and use of the model (e.g., physicians, psychologist, nurses, nurse practitioners, social workers, occupational therapists, care co-ordinators) effectively implemented the DMC Model in Edmonton and Calgary zones, and to varying degrees in the other zones from 2010-2012.

Discussion \& Conclusion: The DMC Model offers a holistic inter-disciplinary approach to capacity assessment that maximizes client autonomy, offers the least restrictive and intrusive solutions, and facilitates inter-disciplinary and inter-organization collaboration.

The Accumulation of Health Deficits Predicts Dementia and Mortality in Healthy Individuals

Roxanne Sterniczuk ${ }^{1}$, Olga Theou ${ }^{2}$, Benjamin Rusak ${ }^{1}$, Kenneth Rockwood ${ }^{2}$

${ }^{1}$ Departments of Psychiatry and Neuropsychology, Dalhousie University, Halifax, NS, and ${ }^{2}$ Geriatric Medicine Research, Dalhousie University, Halifax, NS, Canada
Background/Purpose: In a re-analysis of data from the Canadian Study of Health and Aging, non-traditional risk factors, which were not typically associated with dementia, were found to impact an individual's level of frailty and subsequently their risk of Alzheimer's disease (AD). We examined whether an index consisting of such factors could predict future reports of incident $\mathrm{AD}$ and dementia, as well as mortality, in a similar manner to traditional risk factors, in a larger, multinational cohort.

Methods: Secondary analyses were conducted on data from the Survey of Health, Ageing, and Retirement in Europe and consisted of cognitively healthy individuals 50 years or over, from 12 European countries $(\mathrm{N}=11,817)$. Three AD risk factor indices (RFIs) were constructed to predict a $\sim 4$-year risk for a self or informant report of $\mathrm{AD}$, dementia, and survival; a 31-item non-traditional RFI, a 6-item traditional RFI, and a 37-item combined RFI.

Results: After adjusting our risk model for age, sex, education, and traditional risk factors for $\mathrm{AD}$, the non-traditional RFI significantly predicted the risk of dementia $(\mathrm{OR}=$ $1.49,95 \%$ CI $1.34-1.67$ ), and mortality (OR $=1.53,95 \%$ CI 1.19-1.96) after an average of 4.3 years. The combined RFI exhibited the strongest prediction of dementia $(\mathrm{OR}=$ $1.79,95 \%$ CI $1.38-2.32)$ and mortality $(\mathrm{OR}=1.68,95 \% \mathrm{CI}$ $=1.50-1.89$ ).

Discussion \& Conclusion: The typically small impact of health deficits that are not traditionally associated with AD can significantly increase one's risk of both dementia and mortality when combined. Health professionals should place greater importance on the examination of overall health decline, rather than solely assessing traditional risk factors for illness.

\section{A Prospective Study Examining Pros and Other Geriatric Outcomes in Older Adults with Prostate Cancer Undergo- ing Chemotherapy}

Tharsika Manokumar ${ }^{1}$, Salman Aziz ${ }^{1}$, Faraz Rizvi ${ }^{1}$, Henriette Breunis $^{1}$, Anthony Joshua ${ }^{2}$, Ian Tannock ${ }^{2}$, Shabbir Alibhai ${ }^{1}$ ${ }^{1}$ University Health Network, Toronto, ON, and ${ }^{2}$ Division of Medical Oncology and Hematology, Princess Margaret Cancer Centre, Toronto, ON, Canada

Background/Purpose: Based on clinical trials, treatment of metastatic castration-resistant prostate cancer (mCRPC) with chemotherapy is seen to improve disease control and survival in older men (age 65+). Its effects, though, on the daily functioning, physical performance, and quality of life (QOL) in elderly men outside the clinical trial setting are not well understood. 
Methods: Men aged 65+ with mCRPC starting first-line chemotherapy at a tertiary cancer centre were enrolled in this prospective observational pilot study. Physical function was assessed with the timed up and go (TUG) test, Timed Chair Stands, and grip strength. Functional status was measured using the OARS-IADL questionnaire, in addition to social activities limitations and social support (MOS measures). Patients completed the FACT-P and FACT-G to measure prostate-specific QOL and general QOL, respectively. Assessments were completed before each cycle of chemotherapy. Pre-post within-group comparisons were done using Student's $t$-tests and linear regression.

Results: 25 patients (mean age 75 ) receiving Docetaxel + Prednisone were enrolled, 3 of whom died and 2 dropped out. Both general and prostate-specific QOL improved over a median of 6 cycles. Patients' instrumental activities of daily living (IADL) scores remained stable over time. On average, grip strength was stable, and lower extremity function improved on both the TUG and Timed Chair Stands.

Discussion \& Conclusion: Contrary to our hypotheses, QOL improved in this frail elderly cohort, and IADL function remained stable. Although physical function remained stable or improved during first-line chemotherapy, there was significant variability among individual patients. Older men with $\mathrm{mCRPC}$ appear to tolerate first-line chemotherapy fairly well in terms of QOL and geriatric domains.

Early Prognostic Indicators for Delirium in Medically III, Hospitalized Elderly Patients: a Cohort Study

Monidipa Dasgupta, Chris Brymer

University of Western Ontario, London, ON, Canada

Background/Purpose: Despite treatment of the associated condition delirious persons do not always recover, for unknown reasons. We sought to derive and validate a prognostic model to predict poor recovery after an episode of delirium based on early admission characteristics.

Methods: This prospective cohort study consecutively enrolled older medical in-patients (admitted to London Health Sciences Centre) from the community. Participants were screened for delirium. Delirious (by the Confusion Assessment Method) patients were followed in hospital and after discharge.The primary outcome was poor recovery, in delirious patients, defined by death, institutionalization or functional decline (decreased activities of daily living), at discharge or 3 months after discharge, elicited from the medical chart or post-discharge caregiver telephone interviews.

Results: 1235 medical in-patients (mean age 82.6 years, $42 \%$ male) were screened. Delirium occurred in 355 (or
$29 \%$ ) and recovery status was known in 342 (96\%). Fiftyfour patients $(15 \%)$ died in hospital and $24 \%(n=86)$ were discharged to a permanent residential institution. At a median of 103 days after discharge, another 97 (or 48\%) delirious individuals who were discharged from hospital, had poor recovery (one deceased, 50 institutionalized, and 46 with decreased activities of daily living ability), resulting in an overall rate of poor recovery of $69 \%$ (237). Poor recovery was associated with advanced age, lower baseline function, not being on a benzodiazepine prior to admission, hypoxia, having higher delirium severity scores, and acute renal failure. This model was predictive of poor recovery in the validation sample (ROC area of $0.68,95 \%$ CI: $0.57-0.80$ ).

Discussion \& Conclusion: Results suggest that poor recovery after delirium is common, and is associated with certain characteristics available on admission.

\section{Gait and Balance Scales Recommended to Standardize Practice in Quebec Geriatric Assessment Units (GAU)}

Thien T. Minh $\mathrm{Vu}^{1}$, Judith Latour ${ }^{2}$, Marie-Jeanne Kergoat ${ }^{3}$, Francois Dube ${ }^{3}$, Aline Bolduc ${ }^{3}$

${ }^{I}$ Centre hospitalier universitaire de Montréal, Montreal, $Q C$; ${ }^{2}$ Centre hospitalier universitaire de Montréal, Montréal, QC, ${ }^{3}$ Institut universitaire de gériatrie de Montréal, Montréal, QC, Canada

Background/Purpose: The Regroupement des Unités de courte durée gériatriques et des services hospitaliers de gériatrie du Québec (RUSHGQ) is a community of practice, established in 2010, bringing together health professionals and managers working in GAU. It was previously observed that the quality of care processes varies between GAU. The mobility committee of the RUSHGQ recommends that all GAU units use similar gait and balance scales to standardize patient evaluation, management and follow-up in Quebec. The objectives of the study are: 1) to characterize scales used by physiotherapists; and 2) to inquire about scales that must be used to assess patients with moderate-to-severe gait and balance disorder.

Methods: Two surveys were held among physiotherapists and physical rehabilitation therapists working at a GAU unit $(n=48)$ associated with the RUSHGQ.

Results: Overall, professionals from $36 \mathrm{GAU}$ responded to one or both surveys. The most frequent scales used by the participants are Berg Balance Scale (BBS)-97\%; Timed Up and Go Test (TUG) $-80 \%$; and walking speed test $-57 \%$. Those tests (BBS, TUG, and walking speed test) were also the most frequently recommended by the participants for assessing a patient with moderate-to-severe gait and balance disorder. 
Discussion \& Conclusion: The mobility committee of the RUSHGQ recommends that the assessment of gait and balance disorders should include at least the Berg Balance Scale, the Timed Up and Go Test, and a walking speed test.

\section{Subjective Cognitive Impairment: a Four-Year Case Finding Study}

Sarah Woolmore-Goodwin ${ }^{1}$, Michael Borrie ${ }^{2}$, Patricia Sargeant ${ }^{3}$, Brittany Lloyd ${ }^{4}$

${ }^{1}$ Lawson Health Research Institute, London, ON; ${ }^{2}$ Department of Medicine, Division of Geriatric Medicine,University of Western Ontario, London, ON; ${ }^{3}$ Geriatric Clinical Trials Group, London, ON; ${ }^{4}$ Interdisciplinary Health Studies, Ottawa, ON, Canada

Background/Purpose: Traditionally physicians have viewed Subjective Cognitive Impairment (SCI) in older people to be benign and related to age-associated memory loss. However, research in this field suggests that people who self-report memory problems, but score normal on cognitive testing, have a higher rate of progressing to mild cognitive impairment (MCI).

Methods: Over the last 4 years a total of 165 people over 55 responded to newspaper advertisements with self-reported memory loss. Participants received cognitive screening tests using the standardized MMSE, the MoCA, the 15-point GDS, the AD8, the Cornell Scale for Depression in Dementia, and the Lawton Brody Activities of Daily Living Scale. The test results were case conferenced with a geriatrician, and a clinical suspicion of normal, SCI, MCI, depressive symptoms/mixed picture, possible dementia or other was given. 46 individuals have repeat measures on these tests from 2009 to 2012 .

Results: In 2012, of those 46 follow-up participants, 54\% had no change on their cognitive tests. However 33\% had declined over the 4 years and $9 \%$ had improved. Of those who were given the clinical impression of SCI in 2009 or $2010,39 \%$ had declined to amnestic MCI or multiple-domain MCI. Those individuals who reported depressive symptoms in 2009 (32\%) tended to have lower scores on the GDS and Cornell on follow-up visits.

Discussion \& Conclusion: In studies published on SCI, those who self-report memory problems compared to normal health controls are at greater risk of declining to MCI.

Our study captured this trend as 39\% of those with SCI had declined to MCI within 4 years.

Those with depressive symptoms may have improved with non-drug/drug approaches.

\section{TRAINEE PODIUM ABSTRACTS}

\section{Approach to Sedative Hypnotic Use Among Older Adults}

Jacqueline McMillan ${ }^{1}$, Jayna Holroyd-Leduc ${ }^{1}$, Elizabeth Aitken $^{2}$

${ }^{I}$ Department of Geriatrics, University of Calgary, Calgary, $A B$, and ${ }^{2}$ Department of Health Sciences, University of Calgary, Calgary, AB, Canada

Background/Purpose: Many older adults are prescribed benzodiazepines despite their association with cognitive decline, postural instability, falls, hip fractures, and a five-fold risk of hospitalization after a motor vehicle collision. Yet, 16\% to $33 \%$ of elderly, community-dwellers use benzodiazepines, and $54 \%$ use them daily. In this review, we address the approach to discontinuation and effective alternative options.

Methods: MEDLINE (1946-2012), EMBASE (1980-2012), and the Cochrane Database of Systematic Reviews (20052012) were searched. The following key search terms were used: MeSH \& EMBASE terms for benzodiazepines, sleep initiation and maintenance disorders, drug withdrawal and abuse terms, and keywords for sleep, addiction, dependence, and insomnia, as well as specific drug names and terms for taper, withdrawal, and alternative therapies.

Results: Chronic benzodiazepine use is associated with many adverse outcomes. Hospitalization may play a pivotal role in both the initiation and discontinuation of sedative hypnotics. There is a paucity of long-term data for the use of non-benzodiazepine sedative hypnotics. Cognitive behavioural therapy, brief behavioural interventions, and benzodiazepine tapering protocols have shown proven benefit in benzodiazepine discontinuation.

Discussion \& Conclusion: There may be evidence for non-benzodiazepine sedative hypnotics; however, there is a paucity of long-term, placebo-controlled studies to support their safety, and some evidence to suggest harm in the frail older adult. Cognitive behavioural therapy and/or the use of a taper protocol may increase the success of withdrawal and improve sleep parameters. Exercise, sleep education, massage, and brief behavioural intervention are excellent non-pharmacological options for managing insomnia and for aiding discontinuation. Lastly, it is important to be cognizant 
of the impact that prescribing sedative hypnotics in hospital can have on long-term use.

\section{Time Requirements of Chronic Disease Guidelines in Primary Care}

Jason Kerr ${ }^{1}$, Sharon Straus ${ }^{1}$, Nav Persaud ${ }^{2}$

${ }^{1}$ Geriatric Medicine, St. Michael's Hospital, Toronto, ON, and ${ }^{2}$ Family and Community Medicine, St. Michael's Hospital, Toronto, ON, Canada

Background/Purpose: Clinical practice guidelines are intended to improve patient care. Clinicians may not be able to implement guideline recommendations because of time pressures, which are particularly challenging in primary care. We aimed to quantify the time required to implement guideline recommendations regarding the most common chronic diseases in older adults, including hypertension, diabetes, dyslipidemia, asthma, chronic obstructive pulmonary disease, and chronic kidney disease.

Methods: We determined the time required to apply national guidelines to a cohort of primary care patients. Eight Canadian clinical practice guidelines addressing management of chronic diseases in adults were reviewed. Their recommended interventions, along with the indications for each intervention, were identified. Three primary care physicians reviewed each recommendation and identified the time required to perform it on an average patient. A cohort of 160 randomly selected patients aged 55 years from a university-affiliated primary care clinic was analyzed to determine how often each intervention should be applied to these patients. These data were used to estimate how much time it would take a clinician to apply guideline recommendations to his or her practice.

Results: 103 different interventions from 8 clinical practice guidelines were identified. The total time required to apply these interventions to the selected cohort of patients was 340 hours ( $\mathrm{SD} \pm 189$ ). Extrapolating this value to a clinical roster of 1000 patients, 266 working days would be required each year to implement the recommended interventions.

Discussion \& Conclusion: The implementation of chronic disease guideline recommendations in primary care requires a prohibitive amount of time. Guideline developers should consider the time required to implement their recommendations when drafting clinical practice guidelines.

Development of a Standardized Tool for Evaluation and Development of the Framework and Processes of Care in GEMU
Émilie Breton ${ }^{1}$, Stéphane Lemire

${ }^{I}$ Département de médecine interne, Université Laval, $Q C$; ${ }^{2}$ Centre d'Excellence sur le Vieillissement de $Q C, Q C$, Canada

Background/Purpose: Quebec will face accelerated aging of its population in the years to come. Its health-care system will have to adapt to this situation in order to assure efficiency and relevance of interventions to meet the growing needs. The model of care of the Geriatric Evaluation and Management Unit (GEMU) is a well-known hospital-based mode of organization of geriatric services, and its efficiency has been proven. However, the implementation of this model of care within various Quebec hospitals has brought a noticeable heterogeneity in the care practices among GEMUs. We then want to provide hospital managers with a tool which would define the processes and framework needed to efficiently run GEMUs. This tool would direct the evaluation and development of these services with a strong scientific basis.

Methods: We first did a worldwide literature review and identified two recent meta-analyses on the efficiency of GEMUs. The studies included in the two meta-analyses were rigorously selected and both were analyzed. We also included in our review a Quebec Delphi study on selection criteria applicable to the GEMUs in Quebec.

Results: We extracted and categorized all the process of care items from the studies including: patient selection, type of ward, type of health centre, composition of the geriatric team, and evaluation and treatment processes.

Discussion \& Conclusion: This tool will allow the decision makers and hospital managers to conduct evaluation and development of GEMUs in Quebec and elsewhere.

\section{Reasons and Outcomes for Long-Term Care Transfers to Hospital: a Retrospective Chart Review}

Amanda Gardhouse ${ }^{1}$, Sophie Corriveau', Daniel BrandtVegas $^{1}$, Nidhi Kumar Tyagi ${ }^{1}$, Timothy O'Shea ${ }^{2}$

${ }^{1}$ Internal Medicine, McMaster University, Hamilton, ON, and ${ }^{2}$ Department of Infectious Disease \& Internal Medicine, McMaster University, Hamilton, ON, Canada

Background/Purpose: Studies have shown increased adverse outcomes are related to hospital admissions from Long-Term Care (LTC) homes, often for etiologies that could be safely treated in the facility. We examined the reasons for transfer and outcomes of LTC residents admitted to Hamilton Health Sciences (HHS) hospitals.

Methods: Patient matched hospital and LTC home charts were retrospectively reviewed for all HHS hospital admissions 
transferred from LTC homes during 4 non-consecutive months in 2011. We considered patient demographics, events leading to transfer, diagnosis, and course during admission to hospital. Data presented within are limited to the analysis of hospital medical charts.

Results: A total of 201 charts were reviewed. Altered level of consciousness (21\%), dyspnea (18\%), and fever (9\%) were the most frequent events leading to transfers from LTC homes. Most patients (33\%) transferred for altered LOC were diagnosed with either a urinary tract infection (UTI) or pneumonia. A total of 47 patients experienced an adverse event(s) while hospitalized. Fifteen patients were transferred despite a "do not hospitalize" order. Advanced directives were not documented in 34 patients on arrival to the hospital.

Discussion \& Conclusion: The rate of adverse events in patients transferred from LTC homes to hospitals is high. An intervention aimed at identifying early signs of altered level of consciousness, as well as treating frequent causes, such as UTI's and pneumonia in the LTC homes, may prevent avoidable transfers to hospitals. There is a need to improve discussions and documentation of advanced directives, as well as a system to ensure these are followed.

\section{Hyponatremia in Older Medical Patients: Implications for Adverse Outcomes}

Javier Torres ${ }^{1}$, Shabana Ahamed ${ }^{1}$, Binara Jayasinghe ${ }^{1}$, Kerrie Sanders ${ }^{2}$, Mahesan Anpalahan ${ }^{3}$, Edward Janus ${ }^{1}$

${ }^{I}$ General Internal Medicine, Western Hospital, Melbourne, Victoria; ${ }^{2}$ North West Academic Centre, Melbourne, Victoria; ${ }^{3}$ General Internal Medicine, Geriatrics, Renal Medicine and Metabolic Bone Disorders, Western Hospital, Melbourne, Victoria, Australia

Background/Purpose: Hyponatremia has been associated with increased mortality and length of stay (LOS) in hospitalized patients. However, other adverse associations such as falls or syncope, fractures, unplanned readmission, need for inpatient rehabilitation, and change in discharge destination to a more dependent category have not been widely studied. Our aim was to investigate these associations.

Methods: This is a retrospective case control study of patients admitted with hyponatremia (serum $\mathrm{Na} \leq 134 \mathrm{mEq} / \mathrm{l}$ ) under the General Internal Medicine Unit during a 6-month period. The relevant data were collected by explicit medical record review and analyzed in univariate and multivariate models. Data from 3 months in patients aged 65 years are presented.

Results: The prevalence of hyponatremia was $21 \%$. Hyponatremia had a significant univariate association with LOS
(OR $1.03 p=.016$ ), unplanned readmission within 30 days (OR 2.43, $p=.017$ ), falls or syncope at presentation (OR 4.0, $p<.001$ ), and admission diagnosis of metabolic disorders (OR 17.27, $p<.001$ ). However, after adjustments hyponatremia was independently associated with only unplanned readmission within 30 days (OR3.0, CI: 1.4, 6.6; $p=.005$ ), falls or syncope (OR 4.4, CI: 2.2, 9.0; $p \leq .001$ ), and admission diagnosis of metabolic disorders (OR13.7, CI: 3.1, 60.0; $p=.001)$. Although other predefined adverse associations more frequently occurred in hyponatremic patients, they were not significant.

Discussion \& Conclusion: The study confirms the association between hyponatremia and falls or syncope. Among the adverse outcomes of hospitalization, hyponatremia was independently associated with only unplanned readmission within 30 days. Falls or syncope at presentation and admission diagnosis of metabolic disorders appear to have a greater association with LOS than hyponatremia. The study was probably underpowered to assess other outcomes.

\section{Care of the Elderly Training in a Family Medicine Residency Program: an Evaluation Based on Residents' Perceived Needs}

Susan Mercer

Faculty of Medicine and Dentistry, Division of Care of the Elderly, University of Alberta, Edmonton, AB, Canada

Background/Purpose: In 2011, the Memorial University Family Medicine (FM) Residency Program introduced a Care of the Elderly (COE) rotation to enhance residents' skills in managing the complex health issues of the elderly population. The purpose of this project was to understand FM residents' perceived needs in $\mathrm{COE}$ training and to evaluate the $\mathrm{COE}$ rotation with respect to these needs.

Methods: Survey methodology was used with the prerotation survey designed to evaluate perceived needs in $\mathrm{COE}$ training and the post-rotation survey designed to assess whether learning needs where addressed.

Results: The pre-rotation survey was sent to 57 FM residents with a response rate of $40 \%$. The majority of students indicated a need for further training in COE topics. Students identified that in certain areas further training was necessary or essential. These included managing polypharmacy $(65.2 \%$ identified this as essential), managing the behavioural and psychological symptoms of dementia (52.2\% as very necessary and $39.1 \%$ as essential), and managing chronic wounds (65.2\% as very necessary). The post-rotation survey was sent to $11 \mathrm{FM}$ residents with a response rate of $82 \%$. Most students felt learning needs were fully satisfied in the following areas: 
performing a dementia assessment (55.6\%); distinguishing between dementia, delirium and depression (55.6\%); and managing the behavioural and psychological symptoms of dementia (66.7\%).

Discussion \& Conclusion: Memorial University FM residents recognize the need for $\mathrm{COE}$ training. With the growth of the elderly population, newly trained family physicians must be prepared to provide these patients with appropriate care. This COE rotation addresses most learning needs. However, results from our survey indicate that there is room for improvement.

\section{Comparison of the Test Your Memory Self-Administered Cognitive Screening Test to the Mini-Mental State Exam in a Primary Care Setting}

Karenn Chan ${ }^{1}$, Keith Wilson ${ }^{2}$, Carl Hudson ${ }^{2}$, Vaughn Smith ${ }^{2}$ ${ }^{I}$ Care of the Elderly, University of Alberta, Edmonton, AB, and ${ }^{2}$ Family Medicine, Dalhousie University, Saint John, $N B$, Canada

Background/Purpose: The most common cognitive screening tool used by family physicians is the Folstein Mini-Mental State Examination (MMSE). In 2009, Brown et al. created a new cognitive screening test called the Test Your Memory (TYM), which is unique in the fact that it is a patient self-administered exam. In a system where family physicians and other specialists are pressed for time, the TYM offers a potential to save 10 minutes of screening time. This study aimed to determine the validity of the TYM tool in comparison to the traditional MMSE in a Canadian primary care sitting.

Methods: Patients aged 65 and older attending a regularly scheduled appointment in two family physician offices in New Brunswick were invited to participate in the study. Participants had to complete the self-administered Test Your Memory tool and complete a MMSE.

Results: A total of 52 participants completed the study. The mean TYM score was 44.7/50 (SD 2.4) and the mean MMSE score was 27.8 (SD 5.6). The Pearson correlation coefficient between the TYM and MMSE is $\mathrm{R}^{2}=.58$. This is a significant correlation with a p-value of .01. A score of $\leq 42 / 50$ on the TYM had a $100 \%$ specificity for picking up patients who will score $<24$ on the MMSE. The sensitivity of the TYM was $100 \%$ and the specificity was $81.6 \%$.

Discussion \& Conclusion: This study validates the TYM test as a screening tool in a Canadian primary care population. However, the strength of the TYM test is in its negative predictive value in participants who score above 42 .
Accelerometer-Based Measures of Sedentary Behaviour and Cardio-Metabolic Risk Factors in Active Community-Dwelling Older Adults

Jocelyn Chase, Chris Lockhart, Maureen Ashe, Graydon Meneilly, Kenneth Madden

Division of Geriatric Medicine, VITALiTY (Vancouver Initiative to Add Life to Years) Research Centre, University of British Columbia, Vancouver, BC, Canada

Background/Purpose: Sedentary behaviour has been proposed as an independent cardiometabolic risk factor, even in adults who are otherwise physically active through leisure-time recreational activities. Because little is known about the metabolic effects of sedentary behaviour in seniors, we examined the relationship between sedentary behaviour and cardiometabolic risk in physically active older adults.

Methods: 54 community-dwelling men and women 65 years of age (mean 71.5 years) were enrolled in this crosssectional observational study. Subjects were in good health and free of known diabetes. Activity levels (sedentary, light activity, moderate activity, and vigorous activity time per day) were recorded with accelerometers worn continuously for 7 days. Cardiometabolic risk factors measured consisted of the American Heart Association diagnostic criteria for metabolic syndrome (waist circumference, triglycerides, high-density lipoprotein (HDL), systolic blood pressure, fasting glucose), as well as low-density lipoprotein (LDL). The relationships between activity measures and cardiometabolic risk factors were examined. Significant variables were entered into a multivariate regression model.

Results: All but 1 subject met Canada Health guidelines for an active "fit" adult. Despite this, the average proportion of time spent at a sedentary activity level each day was $72.7 \%$. From the regression analysis, the only significant association found was between LDL and sedentary time, with LDL detrimentally associated with average sedentary time per day (Standardized Beta Correlation Coefficient $0.302, p<.05)$.

Discussion \& Conclusion: Sedentary behaviour is associated with an adverse metabolic effect on LDL in older adults, even those who meet Canada Health guidelines for an active "fit" adult. Emphasizing activities that reduce sitting (e.g., standing desks, less television) may be a practical recommendation to reduce sedentary behaviour in older adults.

Antipsychotics for the Prevention of Post-Operative Delirium: a Meta-Analysis 
Mark Fok ${ }^{1}$, Amir Sepehry ${ }^{2}$, Larry Frisch ${ }^{3}$, Peter Chan $^{4}$, Sharon Strauss ${ }^{5}$, Richard Sztramko ${ }^{6}$

${ }^{1}$ University of British Columbia, Vancouver, BC; ${ }^{2}$ College for Interdisciplinary Studies, Graduate Program in Neuroscience, University of British Columbia (UBC), Vancouver, BC; ${ }^{3}$ Vancouver Coastal Health Research Institute, Vancouver, $B C ;{ }^{4}$ Department of Psychiatry, Vancouver General Hospital, Vancouver, BC; ${ }^{5}$ Keenan Research Centre of the Li Ka Shing Knowledge Institute of St. Michael's Hospital, Toronto, ON; ${ }^{6}$ Division of Geriatrics, Department of Medicine, St. Paul's Hospital, Vancouver, BC, Canada

Background/Purpose: Post-operative delirium in older adults is a common complication of surgery with significant consequences. Delirium often portends poorer clinical outcomes including increased mortality, length of stay, and increased likelihood of discharge to a facility. The role of antipsychotics to prevent post-operative delirium has not been well-established. We therefore wished to determine the effectiveness of antipsychotics in preventing postoperative delirium.

Methods: We searched online literature databases and registers for randomized controlled trials (RCTs) of adults undergoing surgery who were given antipsychotics to prevent post-operative delirium, using a placebo as the comparator. Two researchers independently reviewed citations and abstracts, selecting those meeting inclusion criteria. Quality was assessed via the Cochrane risk of bias tool. Random effects meta-analysis and meta-regression were conducted. Q-statistics and $\mathrm{I}^{2}$ were used for assessment of heterogeneity.

Results: We evaluated 4340 citations from our initial search and from this reviewed 32 full-text articles. Five randomized controlled trials met criteria for inclusion. Antipsychotics were found to reduce post-operative delirium [OR: 0.41; 95\% CI: 0.235 to 0.744$]$. The effect-size estimate was heterogeneous [Q-value: $\left.15 ; p=.003 ; \mathrm{I}^{2}=75\right]$ and overall significant $[p=.003]$. Further examination of the heterogeneity showed that several factors could help reach statistical homogeneity: acuity of surgery (elective vs. mixed acute/elective), antipsychotic type (generation), and method of administration. Meta-regression showed that as one gets older and as the dosage in chlorpromazine equivalents increases, the Log Odds Ratio increases.

Discussion \& Conclusion: Within the limits of few RCT's available, antipsychotics appeared to reduce the incidence of post-operative delirium in a variety of surgical settings. Larger, well-designed RCTs are needed to help confirm our findings.

The Cognitive Profile of PiB-Positive (PiB+) and PiBNegative (PiB-) Subjects with Mild Cognitive Impairment
Elise Levinoff ${ }^{1}$, Natalie Phillips ${ }^{2}$, Howard Cherktow ${ }^{1}$, Victor Whitehead $^{1}$

${ }^{1}$ McGill University, Montreal, $Q C$, and ${ }^{2}$ Department of Psychology, Concordia University, Montreal, QC, Canada

Background/Purpose: Patients with mild cognitive impairment (MCI) and significant amyloid burden on PiB PET imaging manifest impaired performance on episodic memory tasks when compared to MCI patients with lower amyloid burden. This association has yet to be defined with regards to non-episodic memory tasks. Therefore, we sought to further characterize the cognitive profile of subjects with MCI who underwent PiB PET imaging.

Methods: Forty-six subjects aged 60-90 with a clinical diagnosis of MCI underwent neurospychological evaluation. PiB PET images were obtained within 8 months of a subject's cognitive assessment. Subjects were matched for age and education and classified as $\mathrm{PiB}-(\mathrm{SUV}<1.5 ; \mathrm{n}=22)$ or $\mathrm{PiB}+$ $(\mathrm{SUV}>1.5 ; \mathrm{n}=24)$. The results from the neuropsychological evaluation were compared between groups and correlated with amyloid burden. A regression analysis was conducted to determine whether amyloid burden was a predictor of cognitive performance.

Results: There were no significant group differences on global cognitive measures. There was considerable overlap between $\mathrm{PiB}+$ and $\mathrm{PiB}-$ subjects on all cognitive domains, but the $\mathrm{PiB}+$ subjects performed significantly worse than PiB- subjects on tasks of episodic memory and executive functioning. Regression analysis showed that amyloid beta deposition was a significant predictor of performance on episodic memory and inhibition.

Discussion \& Conclusion: These preliminary results suggest that MCI patients who are considered to be prodromal Alzheimer's disease may be distinguishable by the presence of impairment in both episodic memory and inhibition. Future studies may be useful for addressing whether a specific neuropsychological battery can aid in early diagnosis of dementia.

\section{Post-Discharge Medication Errors in Community- Dwelling Frail Older Adults}

Shirley Chien-Chieh Huang ${ }^{1}$, Robyn Savage ${ }^{2}$, Joy Liao ${ }^{2}$ ${ }^{I}$ Department of Medicine, University of British Columbia, Division of Geriatrics, Vancouver, BC, and ${ }^{2}$ Geriatrics Medicine, Mount Saint Joseph Hospital, Vancouver, BC, Canada

Background/Purpose: Frail elderly adults are particularly vulnerable to medication errors when transitioning from hospital to home. The objective of this study is to describe the prevalence and causes of medication discrepancies 
(MDs) in geriatric community-dwelling adults during this transition period.

Methods: A descriptive study was carried out from a community hospital setting in British Columbia, Canada. The study population consists of patients 70 years and older who met selection criteria for home visits within 24-72 hours after hospital discharge by a Geriatric Transition Nurse (GTN) between November 2011 to May 2012. Using the Medication Discrepancy Tool, the GTN performed medication reconciliation between discharge medications and medications individuals were taking at home. Patient-level and system-level factors contributing to the MDs were identified.

Results: Out of the 100 patients seen by the GTN, 65\% were female and $85 \%$ were on five or more medications at the time of discharge. $72 \%$ of patients had five or more comorbid chronic conditions. Medication reconciliation identified $46 \%$ of patients with at least one medication discrepancy. More than half of MDs were caused by patientlevel factors and the remainder were caused by system-level factors. The most common reported patient-level factors were: non-intentional non-adherence and intentional nonadherence. The most frequently seen system-level factors were: incomplete/inaccurate/illegible discharge instructions and not recognizing patient's lack of support. In some instances both types of factors contributed to the occurrence of a medication discrepancy.

Discussion \& Conclusion: Medication discrepancies in the frail elderly are common when transitioning from hospital to home. Identifying common patient-level and system-level factors may serve as starting points when designing quality improvement efforts with the aim to decrease medication discrepancies.

\section{Adapting Osteoporosis Guidelines for Frail Elderly Us- ing GRADE}

Nancy Santesso ${ }^{1}$, Amy Maher ${ }^{2}$, Laura Pickard ${ }^{2}$, Carly Skidmore $^{2}$, Alexandra Papaioannou ${ }^{2}$, Holger Schunemann ${ }^{1}$ ${ }^{I}$ Department of Clinical Epidemiology and Biostatistics, McMaster University, Hamilton, ON, and ${ }^{2}$ Department of Medicine, Geriatrics, McMaster University, Hamilton, ON, Canada

Background/Purpose: In 2010, Osteoporosis Canada developed guidelines for the diagnosis and management of osteoporosis for people $>50$ at high risk of fragility fractures. These guidelines did not address frail elderly where access to diagnostic technology, such as bone mineral density, and research is limited.

Methods: We used the GRADE process to develop guidelines applicable to frail elderly with over 50 stakeholders, including resident/family representatives of long-term care, interdisciplinary health professionals, and program managers. We surveyed the panel to determine questions and outcomes most relevant for this population. We searched the literature for baseline risks of fractures and intervention effects. When making recommendations, we discussed benefits/harms, strength of evidence, values/preferences, and resources.

Results: In addition to outcomes from the 2010 guidelines, this panel identified mobility, pain, and quality of life as important in this population. However, few studies reported these outcomes. To make recommendations, the panel considered absolute risk differences in outcomes with or without treatment, which are calculated from baseline risks. It was critical that the panel agreed on baseline risks which can vary between low- and high-risk groups. Agreement was challenging, but the process was enlightening to recognize gaps/uncertainties in existing research. When evidence in frail elderly was lacking, the panel assessed the applicability of effects found in other populations to make recommendations. The GRADE process incorporated values/preferences, particularly of families and residents, which was uniquely challenging in view of life expectancy, multiple co-morbidities, and serious consequences of fractures.

Discussion \& Conclusion: The GRADE process helped identify gaps in the literature for important outcomes, the impact of baseline risks, and the importance of balancing benefits and harms, and their value and consequences in this population.

\section{Uptake of Evidence-Based Osteoporosis Practices in Long-Term Care (LTC)}

Courtney Kennedy ${ }^{1}$, George Ioannidis ${ }^{1}$, Lehana Thabane ${ }^{2}$, Denis O'Donnell ${ }^{3}$, Lora Giangregorio ${ }^{4}$, Jonathan Derek Adachi $^{1}$, Alexandra Papaioannou ${ }^{1}$

${ }^{1}$ Department of Medicine, McMaster University, Hamilton, ON; ${ }^{2}$ Clinical Epidemiology and Biostatistics, McMaster University, Hamilton, ON; ${ }^{3}$ Medical Pharmacies Group Limited, Pickering, ON; ${ }^{4}$ Department of Kinesiology, University of Waterloo, Waterloo, ON, Canada

Background/Purpose: Since 2006, the Ontario Osteoporosis Strategy for Long-Term Care has engaged in outreach activities to increase uptake of evidence-based osteoporosis/fracture prevention strategies (www.osteoporosislongtermcare.ca). A baseline environmental scan revealed a wide spectrum of prescribing practices between LTC homes reflecting the lack of standardized guidelines and academic detailing. The objective of the present study was to describe current osteoporosis prescribing practices across Ontario LTC homes. 
Methods: In August 2012, de-identified medication/demographic data were downloaded from Medical Pharmacies, a pharmacy provider for approximately one-third of Ontario LTC homes. After excluding 40 LTC homes participating in a targeted intervention (ViDOS), we analyzed data for 166 LTC homes. The percentage of residents receiving 1) Vitamin D ( $800 \mathrm{IU} /$ day), 2) calcium ( $500 \mathrm{mg} /$ day), and 3) osteoporosis medication was calculated for each LTC home. Mean $(95 \% \mathrm{CI})$ LTC home prescribing rates and ranges are reported.

Results: The analysis cohort was 21,699 residents, mean age 83.5 (SD: 10.7) years, $70 \%$ women. $57 \%$ of LTC homes were for-profit, $45 \%$ affiliated with a corporate chain, $61 \%$ had age100 residents. Mean LTC home prescribing rates were 59.9\% (95\% CI: 57.2, 62.6) for vitamin D, 32.2\% (95\% CI: 30.2, 34.2) for calcium, and 18.5\% (95\% CI: 17.4, 19.7) for osteoporosis medications. Prescribing rates were normally distributed and ranged from $22.3 \%-94.9 \%$ (vitamin D), 1.6\%-78.4\% (calcium), and $0 \%-55.9 \%$ (osteoporosis medications).

Discussion \& Conclusion: Although there was a range in prescribing between LTC homes, our results indicate that wide-scale implementation of outreach activities resulted in uptake by many LTC homes, particularly for Vitamin D, with half the homes prescribing at approximately $60 \%$ or better. Currently, osteoporosis consensus guidelines for LTC are being developed.

An Educational Intervention Study to Reduce the Use of Potentially Inappropriate Medications Among Older Adults (EMPOWER Study): Preliminary Effects on Participants' Knowledge, Beliefs, and Risk Perceptions About Benzodiazepine Use

Philippe Martin, Cara Tannenbaum

Institut Universitaire de Gériatrie de Montréal, Montreal, QC, Canada

Background/Purpose: Currently far too many seniors ( 20\%) consume inappropriate benzodiazepines, which increase the risk of adverse drug reactions and unnecessary hospitalizations among community-dwelling elders. As of 2012, the new Beers criteria lists all benzodiazepines as drugs to avoid in the elderly no matter the half-life.

Methods: A written educational tool was mailed to 144 benzodiazepine consumers aged 65 years recruited from community pharmacies. Knowledge and beliefs about inappropriate prescriptions were queried prior to and 1-week after the intervention. Primary outcome was a change in risk perception. Explanatory variables were a change in knowledge and beliefs about medications, as well as cognitive dissonance occurrence. Self-efficacy for tapering and intent to discuss discontinuation were also measured.

Results: Post-intervention, 65 (45.1\%) of chronic benzodiazepine consumers (mean duration use 10.5 years, SD 8.2 years) perceived increased risk. Increased risk perceptions were explained by better knowledge acquisition (mean change score $0.9,95 \% \mathrm{CI}(0.5,1.3))$, and a change in beliefs (BMQ differential mean change score $-5.03,95 \%$ CI $(-6.4,-3.6)$, suggesting elicitation of cognitive dissonance. Experience of cognitive dissonance was associated with a 6-fold higher likelihood of patients reporting increased risk perception $(\mathrm{OR}=6.6195 \% \mathrm{CI}(3.2,13.8))$. Intent to discuss discontinuation of benzodiazepines with a doctor $(83.1 \%$ vs. $44.3 \%, p<.001)$ was higher among participants who perceived increased risk.

Discussion \& Conclusion: Risk perception on benzodiazepines can be altered through direct delivery of an educational tool to aging consumers. Results suggest patients could potentially be targeted directly with information to catalyze discontinuation of inappropriate prescriptions.

Is There a Motor Signature in Mild Cognitive Impairment? Results from the Gait and Brain Study

Ivan Anton-Rodrigo ${ }^{1}$, Karen Gopaul ${ }^{1}$, Mark Speechley ${ }^{2}$, Vladimir Hachinsky ${ }^{3}$, Susan Muir ${ }^{1}$, Anam Islam ${ }^{4}$, Manuel Montero Odasso ${ }^{5}$

${ }^{1}$ Gait \& Brain Lab, Department of Geriatric Medicine, Parkwood Hospital, London, ON; ${ }^{2}$ Department of Epidemiology $\&$ Biostatistics, University of Western ON, London, ON; ${ }^{3} \mathrm{De}$ partment of Clinical Neurosciences, Division of Neurology, London Health Sciences Centre, London, ON; ${ }^{4}$ Department of Geriatric Medicine, London, ON; ${ }^{5}$ University of Western Ontario, London, ON, Canada

Background/Purpose: Gait and cognition are interrelated. Executive dysfunction is associated with slower gait. It is unknown if memory dysfunction, a cardinal sign in MCI, is associated with the gait disturbances seen in MCI. The objective was to determine if gait in older adults with $\mathrm{MCI}$ varies by subtype: amnestic (a-MCI) or non-amnestic (na-MCI) type.

Methods: Older adults with MCI from the "Gait and Brain Study" were included. Cognition was evaluated using MMSE, MoCA, Trails Making Test A and B, Rey Auditory Verbal Learning Test, Digit Span Test, and Letter Number Sequence Test. Gait performance (velocity and gait variability) was evaluated with the GaitRITE $®$ mat under usual walking and three dual-task conditions (walking while: naming animals out loud, serial subtractions by 1s and serial subtractions by 7). Participants were divided into a-MCI and na-MCI by episodic 
memory test. The relationship between cognitive subtype and gait was evaluated with multivariable linear regression.

Results: Fifty-six participants (mean age $76.3 \pm 7.2$ years, $50.9 \%$ female) were included. Thirty-eight were a-MCI and 18 were na-MCI. Groups were similar in age, co-morbidities, and history of previous falls. The a-MCI participants walked slower than na-MCI (98.5 vs. $112.2 \mathrm{~cm} / \mathrm{sec}, p<.03)$ in all test conditions. Regression (adjusted for age, sex, physical activity, number of co-morbidities, and executive function) showed a-MCI was associated with slower gait under usual and dual-task conditions and higher gait variability $(p<.001)$ under dual-task tests.

Discussion \& Conclusion: Episodic memory impairment was associated with poor gait performance, in particular under dual-task conditions. This suggests slow gait and higher variability under dual-task testing is a motor feature in a-MCI independent of executive dysfunction.

\section{Measuring Frailty in Europe: Eight Scales Differ Widely in Identifying the Frail Elderly}

Olga Theou, Thomas D. Brothers, Arnold Mitnitski, Kenneth Rockwood

Geriatric Medicine, Dalhousie University, Halifax, NS, Canada

Background/Purpose: Assessing frailty should be an essential part of the care of older adults. Several scales have been proposed to quantify frailty and the operational criteria of each scale vary. The purpose of this study was to compare the prevalence of frailty in community-dwelling, middle-aged and older Europeans as estimated by eight scales and to examine the agreement among scales in classifying participants as frail.

Methods: 27,527 participants aged 50+ years (mean age $65.3 \pm 10.5,54.8 \%$ women) from the 11 countries (Austria, Belgium, Denmark, France, Germany, Greece, Italy, Netherlands, Spain, Sweden, Switzerland) which participated in the first wave of the Survey of Health, Ageing and Retirement in Europe comprised the study sample. Frailty was operationalized, based on eight scales: frailty phenotype, a 70-item Frailty Index, a 44-item Frailty Index based on a Comprehensive Geriatric Assessment, Clinical Frailty Scale, Edmonton Frail Scale, Groningen Frailty Indicator, Tilburg Frailty Indicator, and "FRAIL" scale. A score threshold was assigned for each scale to represent the frailty state, based on the relevant literature.

Results: The prevalence of frailty ranged from $44 \%$ (Groningen scale) to $6 \%$ (FRAIL scale). About half of participants were categorized differently between scales. $49.3 \%$ of participants were categorized as non-frail by all scales, and $2.5 \%$ were categorized identically as frail by all scales.

Discussion \& Conclusion: Frailty scales capture related but distinct groups of individuals, and each scale provides different estimates of frailty prevalence. Future studies should compare various scales using data from clinical settings.

\section{Evaluation of Osteoporosis Treatment in Hip Fracture Patients Post-Rehabilitation Discharge}

Naomi Dore ${ }^{1}$, Pauline Fisher ${ }^{2}$, Alexandra Papaioannou ${ }^{3}$, Lisa Dolovich ${ }^{3}$, Courtney Kennedy ${ }^{3}$, Jonathan Adachi ${ }^{3}$, Leo Farrauto $^{3}$

${ }^{1}$ Department of Medicine, Hamilton, ON; ${ }^{2}$ Hamilton Health Sciences, Hamilton, ON, ${ }^{3}$ McMaster University, Hamilton, ON, Canada

Background/Purpose: Hip fracture patients are at high risk for recurrence. Appropriate pharmacotherapy reduces this risk and is associated with reduced mortality after hip fracture, but a care gap exists for fracture prevention in these patients. This evaluation determined rates of osteoporosis treatment and bone mineral density (BMD) testing in hip fracture patients following discharge from a rehabilitation unit.

Methods: A prospective cohort study of hip fracture patients aged 50 on an inpatient rehabilitation unit in 2008 and 2011. Patients were seen by a nurse specialist, and encouraged to see their family physician for further assessment and treatment. Physicians were sent a letter indicating the need to follow up with their patient. Patients were contacted following discharge from hospital to determine treatment rates.

Results: Of 310 eligible hip fracture patients admitted to the rehabilitation unit in the years studied, 207 patients were reached post-discharge and provided data. Of patients who were not previously taking osteoporosis medication, $50 \%$ of patients had osteoporosis treatment initiated by 6 months following discharge. By 2 months following discharge, $46 \%$ of patients in the 2008 cohort had a new BMD performed or scheduled, while this was true for $14 \%$ of patients from the 2011 cohort. $35 \%$ of patients in 2011 had not seen their family physician by 2 months following discharge.

Discussion \& Conclusion: Rates for osteoporosis treatment and BMD were higher than those reported in the literature for patients not enrolled in case manager programs. BMD testing declined from 2008 to 2011. Lower treatment rates may be due to concerns regarding bisphophonates. There remains room for improvement for follow-up with family physicians. 
A Pilot Study Comparing the Screen for the Identification of the Cognitive Impaired Medically At Risk Driver (SIMARD) to Clinical Impression of Patients with Dementia or Mild Cognitive Impairment

Madelaine Wernham ${ }^{1}$, Pamela Jarrett ${ }^{2}$, Connie Stewart ${ }^{3}$, Elizabeth MacDonald ${ }^{2}$, Donna MacNeil ${ }^{2}$, Cynthia Hobbs ${ }^{2}$ ${ }^{1}$ Dalhousie Medicine NB, Saint John, NB; ${ }^{2}$ Department of Geriatric Medicine, Horizon Health Network, Saint John, NB; ${ }^{3}$ Department of Computer Science and Applied Statistics, University of NB, Saint John, NB, Canada

Background/Purpose: Assessing fitness to drive in patients with dementia is challenging. The SIMARD was developed as a tool to assist with assessing fitness to drive. This study compares the clinical decision made by a geriatrician regarding driving with the score on the SIMARD.

Methods: Patients seen by geriatricians with a diagnosis of dementia or mild cognitive impairment, who had had a SIMARD test completed after the clinical decision regarding driving was made, were included in the sample. Charts were reviewed to gather diagnosis, driving status and history, cognitive and functional information.

Results: Sixty-three patients were identified and 57 met the inclusion criteria. The mean age was 77.07 years. Alzheimer's dementia in 22 (38.6\%) patients was the most common diagnosis. The mean MMSE was 24.85 (SD 3.34) and the MoCA was 19.85 (SD3.58). The mean SIMARD score was 37.16 (SD 19.54). Twenty-four patients had a SIMARD score below 31 , 28 scored between $31-70$, and 5 scored greater than 70 . Of those scoring less than 31,8 patients continued to drive, 3 of whom had passed a driving test performed by the Department of Public Safety of New Brunswick. In the 5 patients who scored greater than 70,2 had their licenses revoked by the geriatrician.

Discussion \& Conclusion: There did not appear to be a clear association between the SIMARD score and the clinical decision made by the geriatrician.

Modifiable Behaviours in Elderly Cancer Survivors: a Survey of Smoking, Alcohol Use, and Physical Activity

Chongya Niu ${ }^{1}$, Lawson Eng ${ }^{1}$, Xin Qiu $^{2}$, Xiaowei Shen ${ }^{2}$, Osvaldo Espin-Garcia ${ }^{2}$, Dan Pringle ${ }^{1}$, Mary Mahler ${ }^{1}$, Oleksandr Halytskyy ${ }^{1}$, Rebecca Charow ${ }^{1}$, Christine Lam ${ }^{1}$, Ravi M. Shan ${ }^{1}$, Jodie Villeneuve ${ }^{1}$, Kyoko Tiessen ${ }^{1}$, M. Catherine Brown $^{1}$, Peter Selby ${ }^{3}$, Doris Howell ${ }^{4}$, Jennifer M. Jones ${ }^{4}$, Wei $\mathrm{Xu}^{2}$, Geoffrey Liu ${ }^{1}$, Shabbir M.H. Alibhai ${ }^{5}$
${ }^{1}$ Department of Medical Oncology and Hematology, Princess Margret Hospital, Toronto, ON; ${ }^{2} \mathrm{ON}$ Cancer Institute, Princess Margaret Hospital, Toronto, ON: ${ }^{3}$ Addictions Division, Centre for Addiction and Mental Health, Toronto, ON; ${ }^{4}$ University Health Network-Princess Margaret Hospital, Toronto, ON; ${ }^{5}$ Department of Medicine, University Health Network, University of Toronto, Toronto, ON, Canada

Background/Purpose: Cancer survivorship programs often focus on modifiable behaviours such as smoking and alcohol use and physical activity. Whether these behaviours differ among elderly survivors and whether special considerations should be given to these elderly cancer survivors (age $65+$ ) is unclear.

Methods: 616 adult cancer survivors (23\% elderly) across multiple solid and haematologic malignancies and treatment trajectories were surveyed about smoking, alcohol, physical activity, and attitudes and knowledge about effects of these habits on cancer outcomes. Multivariate logistic regression models evaluated the effect of age on these factors.

Results: $\quad 9.0 \%$ of elderly survivors were current smokers; $35.7 \%$ had been binge drinkers recently or in the past ( 5 or more standard drinks per day for male; 4 or more for female); $24.0 \%$ were not meeting exercise guidelines (150 minutes of moderateto-vigorous intensity activity per week). Compared to younger survivors, elderly were one-third as likely to be current smokers $(p<.0001)$, but twice as likely to be ex-smokers than never smokers $(p<.0001)$. They were half as likely to know how smoking affected cancer treatment $(p=.007)$ or prognosis $(p=.008)$. Elderly were one-third as likely to binge drink $(p<.001)$, twice as likely to perceive alcohol as improving survival $(p=.018)$, and half as likely to receive information about alcohol use $(p=.042)$. Meeting exercise guidelines at diagnosis $(p=.015)$ and improving/maintaining them after treatment $(p=.016)$ were lower in elderly survivors, but perceived benefits/harms of exercise did not differ with age.

Discussion \& Conclusion: Elderly cancer survivors have different smoking, alcohol, and exercise characteristics from younger survivors. Survivorship programs may need to tailor counseling by age group.

Reducing Urinary Catheter Utilization in Older Adult Inpatients Using a Multi-Modal Education Intervention

Richard Norman ${ }^{1}$, Rebecca Ramsden ${ }^{2}$, Leanne Verscheure ${ }^{2}$, Samir Sinha ${ }^{3}$

${ }^{1}$ Faculty of Medicine, University of Toronto, Toronto, ON; ${ }^{2}$ Department of Nursing, Mount Sinai Hospital, Toronto, ON; ${ }^{3}$ Geriatrics Program, Mount Sinai Hospital, Toronto, ON, Canada 
Background/Purpose: Indwelling urinary catheterization is a ubiquitous procedure in the inpatient setting: between $16 \%$ and $25 \%$ of hospitalized patients will receive an indwelling catheter at some point during their stay. While sometimes medically indicated, previous studies have shown that between $21 \%$ and $52 \%$ of catheters are used unnecessarily, exposing patients to significant morbidity and mortality, including increased risk of urinary tract infection and bacteremia. Here we present the results of a multi-modal educational intervention directed at reducing the overuse of catheters in a large teaching hospital.

Methods: The multi-modal intervention targeted nurses and used a variety of approaches to improve catheter use, including small group meetings, educational posters, and modifications to the patient chart. The study patient population included all admitted patients to internal medicine, surgery, and orthopedic surgery, as well as the GIM/ACE Unit from 1 September 2009 to 1 October 2011. Data were structured and analyzed as an interrupted time series using a segmented regression approach.

Results: A total of 14,531 patients, 1,878 of whom were catheterized, were included in this study. A decrease in mean catheter days per patient of between 5.8 and 9.7 days $(p<.01)$ across the wards under study was observed after the intervention. The proportion of patients catheterized decreased by between $0.35 \% /$ month and $0.93 \% /$ month $(p<.01)$; ultimately $\%$ patients catheterized halved from $15 \%$ pre-intervention to $7 \%$ post-intervention. A trend of greater discharges directly home was observed in older (65+) patients.

Discussion \& Conclusion: A multi-modal educational intervention using nurse education and process changes resulted in a significant reduction in catheter days per patient and the proportion of patients catheterized.

Performance-Based Measures of Physical Function and Cognition Differ by Migration Status Among MiddleAged and Older Europeans

Thomas Brothers ${ }^{1}$, Olga Theou ${ }^{1}$, Kenneth Rockwood ${ }^{2}$ ${ }^{1}$ Geriatric Medicine Research, Dalhousie University, Halifax, NS, and ${ }^{2}$ Department of Medicine, Dalhousie University, Halifax, NS, Canada

Background/Purpose: Life course influences on health may be most evident at older ages. In a large sample of middle-aged and older Europeans, we compared grip strength, cognitive performance, and walking speed between nativeborn participants, immigrants who were born in low- and middle-income countries (LMICs), and immigrants who were born in high-income countries (HICs).
Methods: This is a retrospective cohort study of the Survey of Health, Ageing, and Retirement in Europe, including 33,745 participants age $50+$ in 14 countries (mean age $=$ $64.9 \pm 10.2$ years; $54 \%$ women). Four performance-based measures were assessed: grip strength, delayed recall, and verbal fluency were measured in all participants, while walking speed was measured only in individuals age $75+$. Analyses were divided by participants' current residence in either relatively wealthier Northern/Western or relatively poorer Southern/Eastern Europe, and adjusted for age, gender, and education.

Results: About $7 \%$ of participants $(n=2,369)$ were immigrants. In Northern/Western Europe, compared to nativeborn participants, LMIC-born immigrants demonstrated weaker grip strength (mean $32.8 \mathrm{~kg}$ vs. $35.7 \mathrm{~kg}, p<.001$ ), and lower delayed recall ( 3.0 vs. 3.6, $p<.001)$ and verbal fluency scores (16.1 vs. 20.4, $p<.001$ ), but similar walking speed $(0.66 \mathrm{~m} / \mathrm{sec}$ vs. $0.72 \mathrm{~m} / \mathrm{sec}, p=.1)$. HIC-born immigrants demonstrated grip strength $(34.7 \mathrm{~kg})$, delayed recall (3.4), and verbal fluency performance (18.5) lower than nativeborn participants, but higher than LMIC-born immigrants $(p<.001)$. In Southern/Eastern Europe, scores did not differ between groups on any measure.

Discussion \& Conclusion: Middle-aged and older immigrants demonstrated worse physical function and cognitive performance than native-born Europeans in Northern/ Western Europe, but not in Southern/Eastern Europe. Country of birth and current country of residence were each associated with these performance-based measures of age-related health.

\section{Considering Both Non-Traditional and Traditional Risk Factors Improves the Prediction of Coronary Heart Disease Events}

Lindsay Wallace ${ }^{1}$, Olga Theou ${ }^{1}$, Michael Rockwood ${ }^{1}$, Susan Kirkland $^{2}$, Kenneth Rockwood ${ }^{1}$, Daichi Shimbo ${ }^{3}$, Karina Davidson $^{3}$

${ }^{1}$ Department of Medicine, Dalhousie University, Halifax, NS, Canada; ${ }^{2}$ Department of Community Health \& Epidemiology, Dalhousie University, Halifax, NS, Canada, ${ }^{3}$ Department of Medicine, Columbia University, NY, USA

Background/Purpose: The importance of traditional risk factors on prediction of adverse events has been established for many chronic diseases. A recent study demonstrated that even non-traditional risk factors, when considered in consort, predicted dementia similarly to any traditional risk factors. The objective of this study was to investigate contributions of non-traditional risk factors to coronary heart disease (CHD) events. 
Methods: This analysis included community-dwelling adults with no history of CHD ( $\mathrm{n}=2669$, mean age $46.4 \pm 19.1$ years, $48.6 \%$ men) who participated in the 1995 Nova Scotia Health Survey. We constructed 3 risk factor indices (RFIs): 1) a 17-item non-traditional RFI (e.g., sinusitis, arthritis); 2) a 9-item traditional RFI (e.g., hypertension, diabetes); and 3) a combined RFI (all 26 items). Ten-year risks of CHD-related hospitalization and mortality were evaluated.

Results: The non-traditional RFI score was significantly predictive of CHD-related hospitalizations and deaths, even after controlling for the traditional RFI (age and sex adjusted hazard ratio [adj. HR] 1.26; 95\% CI 1.09-1.44). However, including all possible variables in the combined
RFI predicted the highest rate of CHD events (adj. HR 1.55; 1.36-1.76). Considered separately, the traditional and nontraditional RFIs similarly discriminated participants who had CHD events from those who did not (area under receiver operating characteristic curve [AUC] 0.70 , CI 0.67-0.74; vs. $0.69,0.65-0.73)$. When all variables were combined in an index, the AUC was significantly higher (combined RFI = $0.76 ; 0.720 .79$ ).

Discussion \& Conclusion: The accumulation of nontraditional risk factors adds a unique contribution to the prediction of CHD hospitalizations and mortality. This supports the idea that maintenance of general health lowers risk for late-life disease. 